\title{
ISOLTRAP: An on-line Penning trap for mass spectrometry on short-lived nuclides
}

\author{
M. Mukherjee ${ }^{1, a}$, D. Beck ${ }^{1}$, K. Blaum ${ }^{1,2,3, b}$, G. Bollen ${ }^{4}$, J. Dilling ${ }^{5}$, S. George ${ }^{1,2}$, F. Herfurth ${ }^{1}$, A. Herlert H,7, $^{6,7}$ \\ A. Kellerbauer ${ }^{3}$, H.-J. Kluge ${ }^{1,8}$, S. Schwarz ${ }^{4}$, L. Schweikhard ${ }^{7}$, and C. Yazidjian ${ }^{1}$ \\ 1 GSI, Planckstraße 1, 64291 Darmstadt, Germany \\ 2 Institut für Physik, Johannes Gutenberg-Universität, 55099 Mainz, Germany \\ 3 Max-Planck-Institute for Nuclear Physics, PO Box 103980, 69029 Heidelberg, Germany \\ 4 NSCL, Michigan State University, East Lansing, MI 48824-1321, USA \\ 5 TRIUMF, Vancouver, British Columbia V6T 2A3, Canada \\ 6 Department of Physics, CERN, 1211 Genève 23, Switzerland \\ 7 Institut für Physik, Ernst-Moritz-Arndt-Universität, 17487 Greifswald, Germany \\ 8 Physikalisches Institut, Ruprecht-Karls-Universität, 69120 Heidelberg, Germany
}

Received: 10 May 2007 / Revised: 7 December 2007

Published online: 27 February 2008 - (C) Società Italiana di Fisica / Springer-Verlag 2008

Communicated by C. Signorini

\begin{abstract}
ISOLTRAP is a Penning trap mass spectrometer for high-precision mass measurements on short-lived nuclides installed at the on-line isotope separator ISOLDE at CERN. The masses of close to 300 radionuclides have been determined up to now. The applicability of Penning trap mass spectrometry to mass measurements of exotic nuclei has been extended considerably at ISOLTRAP by improving and developing this double Penning trap mass spectrometer over the past two decades. The accurate determination of nuclear binding energies far from stability includes nuclei that are produced at rates less than 100 ions/s and with half-lives well below $100 \mathrm{~ms}$. The mass-resolving power reaches $10^{7}$ corresponding to $10 \mathrm{keV}$ for medium heavy nuclei and the uncertainty of the resulting mass values has been pushed down to below $10^{-8}$. The article describes technical developments achieved since 1996 and the present performance of ISOLTRAP.
\end{abstract}

PACS. 07.75.+h Mass spectrometers - 21.10.Dr Binding energies and masses - 32.10.Bi Atomic masses, mass spectra, abundances, and isotopes - 37.10.Ty Ion trapping

\section{Introduction}

The masses of almost all stable nuclides are very well known, some of them with uncertainties down to $10^{-11}$ [1]. However, the mass accuracy of the unstable short-lived nuclides falls off rapidly as a function of the distance from the valley of stability.

The knowledge of nuclear masses far from stability provides crucial tests of current theoretical descriptions of nuclei, including mass models and mass formulae, the study of the character of nucleonic interactions in the nuclear medium, and the search for the superheavy elements [26 . Deviations of the nuclear binding energies from the smooth trend given by simple liquid-drop models [7] unveil nuclear shell structures [8], mass differences between

\footnotetext{
a Present address: IACS/RCAMOS, 2A\&2B, Raja S. C. Mullick Rd., Kolkata 700032, India.

b e-mail: blaumk@uni-mainz.de
}

the odd and even numbered nuclei show the strength of the pairing force [4], double differences of masses can be used to isolate specific nucleonic interactions [9,10], and strong ground-state deformations show up as deviations from the smooth trend of the two-neutron separation energies between shell closures. Furthermore, the determination of nuclear masses has an impact on the elaboration of models of stellar evolution, for example, the r-process (in the neutron-rich environment of supernovae) or the rp-process (in a hydrogen-rich environment, e.g. an X-ray bursts) [11]. An emerging field of application for high-accuracy mass measurements on radionuclides concerns tests of fundamental symmetries as, for example, the study of superallowed $\beta$-decays to test the unitarity of the Cabibbo-Kobayashi-Maskawa (CKM) quark-mixing matrix $[12,13]$.

Radioactive ions typically delivered by an ISOLtype (Isotope Separator On-Line) facility are in the form of continuous beams of some $10 \mathrm{keV}$ of energy and they 


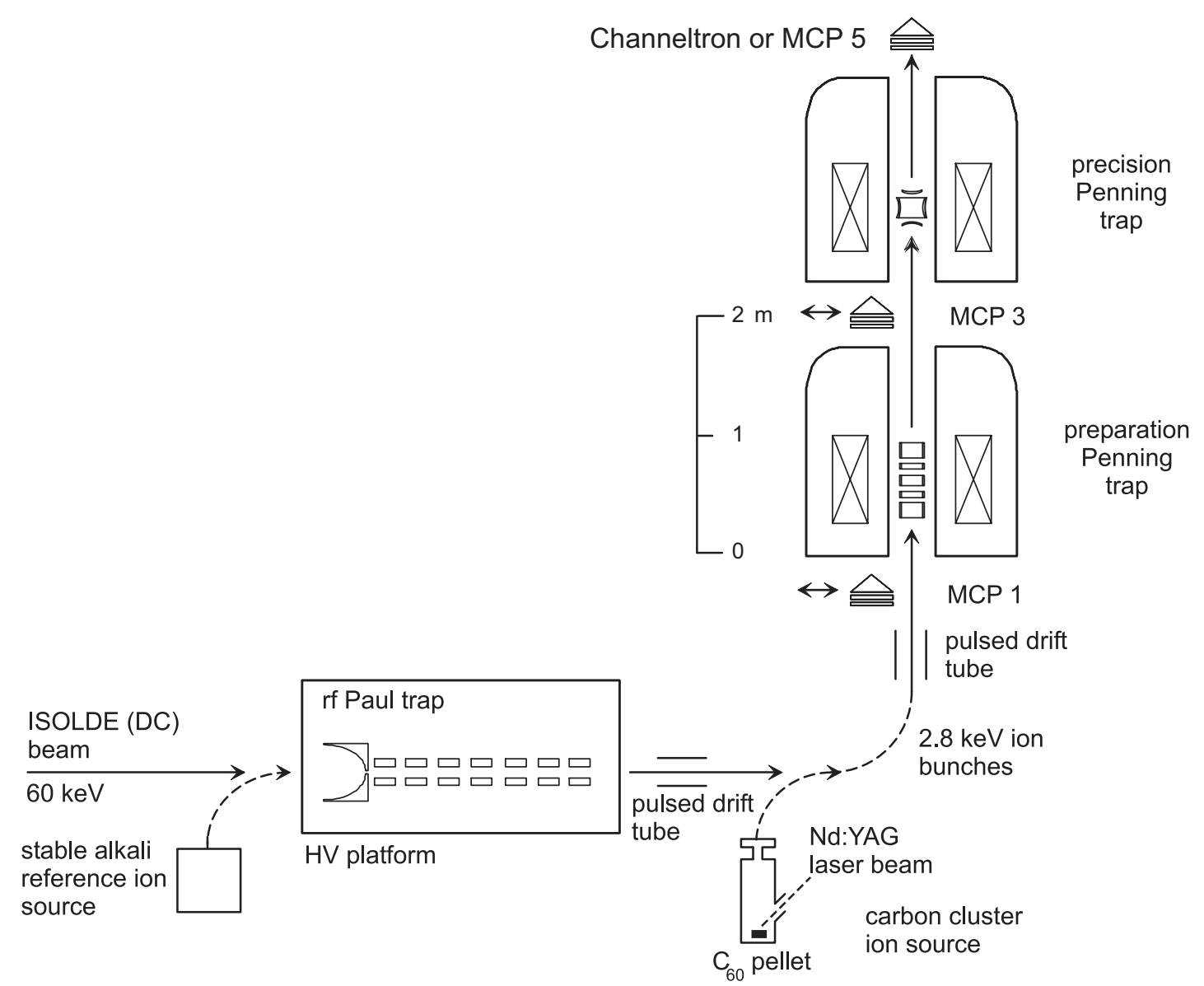

Fig. 1. Schematic layout of ISOLTRAP. The main components are: a linear radiofrequency quadrupole trap used for retardation, cooling, and bunching of the $60 \mathrm{keV}$ ion beam delivered by ISOLDE, a preparation Penning trap for isobaric cleaning of the radioactive ion ensemble, and a high-precision Penning trap mass spectrometer. Micro-channel plate (MCP) detectors are used to monitor the ion transfer as well as to record the time-of-flight ion-cyclotron resonance for the determination of the cyclotron frequency. Recently, a channeltron detector got installed at the position of MCP5 for the time-of-flight measurement to improve the detection efficiency by a factor of about three (for details see text).

span over a wide range of masses. Therefore a highaccuracy mass measurement technique on these unstable nuclei needs to fulfill certain conditions, namely cooling and bunching of the few ten $\mathrm{keV}$ continuous beams, short measurement time, high sensitivity, and versatility to cover a wide range of elements. Many of the techniques needed to fulfill these requirements have been developed at ISOLTRAP over the last two decades (for the status in 1996 see [14], for a more recent brief status report see $[15,16])$. They are now used in almost all Penning trap mass spectrometers installed at a number of radioactive beam facilities in the world $[17,2]$ namely LEBIT at Michigan State University [18], CPT at Argonne National Laboratory [19], JYFLTRAP at the University of Jyväskylä [20], SHIPTRAP at GSI, Darmstadt [21], and TITAN at TRIUMF, Vancouver [22]. These technical innovations include the transfer of ion bunches from one Penning trap into another, i.e. the dynamical capture of ions in a trap [23], the use of radiofrequency azimuthal quadrupolar excitation to measure the cyclotron frequency of radioactive ions stored in a Penning trap [24], the mass selective buffer gas cooling technique [25], the phase-locked magnetron excitation of the stored ions' motion [26], and many more.

In the following the principles used in the ISOLTRAP mass spectrometer, the experimental setup, the mass measurement procedure, and the performance of the present system will be described.

\section{Principles and basic techniques}

The ISOLTRAP mass spectrometer is divided according to functionality into three parts as shown in fig. 1: 1) a linear radiofrequency quadrupole (RFQ) trap [27] for deceleration, cooling, and bunching of the $60 \mathrm{keV}$ ion beam provided by the on-line isotope separator ISOLDE [28]; 2) a gas-filled cylindrical Penning trap for isobaric separation and cleaning of the ion beam via mass selective buffergas cooling [25]; 3) a hyperbolic Penning trap for highprecision cyclotron frequency measurements and mass determinations of the stored ions. 
In the following, the principles and properties of an RFQ trap, of a Penning trap, and the general cooling mechanism in presence of buffer gas will be described as far as they are of relevance here. More detailed descriptions and applications of RFQ traps can be found in [29$36]$ and of Penning traps in $[2,24,37,38]$.

\subsection{Buffer gas cooling}

Buffer gas cooling is conceptually a very simple technique. The (few) stored ions collide elastically with the (many) buffer gas atoms at ambient temperature and thus transfer their kinetic energy to them. After a certain storage time the ions come in thermal equilibrium with the buffer gas atoms.

The cooling mechanism for a large mass difference (the coolant being lighter) and a low relative velocity between the ion and the buffer gas atom is well understood and can be described by considering a viscous damping force proportional to the relative velocity [39]. It is represented as

$$
\boldsymbol{F}=-\delta \cdot m \cdot \boldsymbol{v},
$$

where $m$ is the mass of the ion and $\boldsymbol{v}$ the relative velocity with respect to the buffer gas atom. The damping coefficient of an ion with charge-to-mass ratio $e / m,[40]$

$$
\delta=\frac{e}{m} \cdot \frac{1}{\mu} \cdot \frac{p / p_{\mathrm{N}}}{T / T_{\mathrm{N}}}
$$

is proportional to the buffer gas pressure $p$, inversely proportional to the temperature $T$ and the reduced ion mobility $\mu[40]\left(p_{\mathrm{N}}\right.$ and $T_{\mathrm{N}}$ are normal pressure and temperature of the gas, respectively.) As an example, ${ }^{39} \mathrm{~K}^{+}$ions have in helium buffer gas a mobility of $\mu=2.15 \times 10^{-3} \mathrm{~m}^{2} / \mathrm{V} \mathrm{s}$. Considering a typical buffer gas pressure of $10^{-3} \mathrm{mbar}$ at room temperature, the damping coefficient is $\delta=$ $1100 \mathrm{~s}^{-1}$

\subsection{Beam accumulation in a linear, segmented radiofrequency quadrupole trap}

An ideal quadrupole mass filter, as originally designed by Wolfgang Paul and co-workers [29], consists of four parallel hyperbolic rods where RF voltages are applied to pairs of opposite electrodes. The radial confining force is created by the focusing effect of the ideal quadrupolar field varying with time. Furthermore, ions can be trapped in the axial direction of the device by use of longitudinally segmented rods and a DC trapping potential applied to the segments, as shown in fig. 2. As an example, a singly charged ion of mass $39 \mathrm{u}\left(\mathrm{K}^{+}\right)$confined in an RFQ structure with $r_{0}=6 \mathrm{~mm}, \nu_{\mathrm{RF}}=1 \mathrm{MHz}$, and $U_{\mathrm{RF}}=80 \mathrm{~V}$ will experience a radial trapping potential depth of about $11 \mathrm{~V}$ and oscillates at $\nu_{\mathrm{m}}=196 \mathrm{kHz}$. While being trapped, the ions can also be cooled by collisions with an inert buffer gas like helium. Such an RFQ cooler and buncher has been developed at ISOLTRAP and was installed in the year 2000

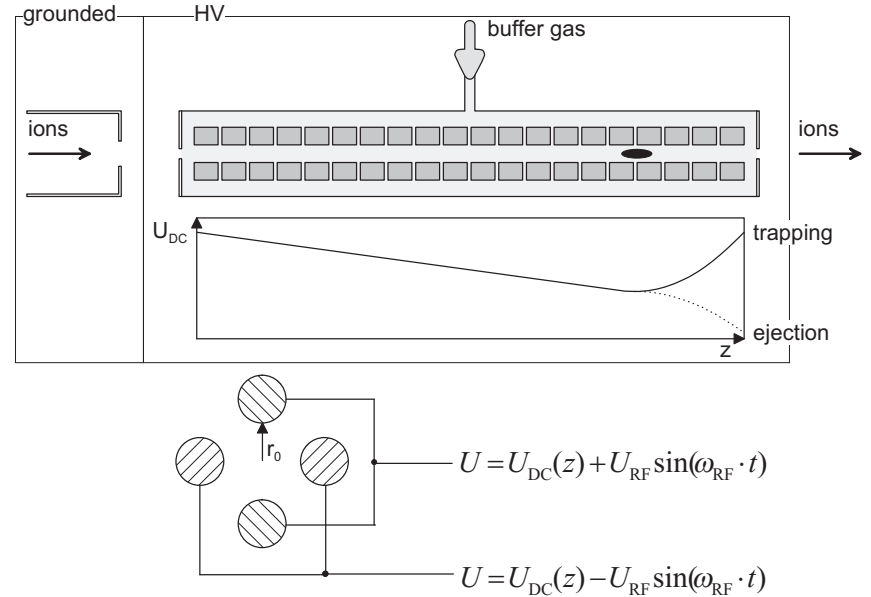

Fig. 2. Sketch of the linear segmented radiofrequency quadrupole trap of ISOLTRAP used as ion beam cooler and buncher. Upper part: schematic side view together with the electric potential along the symmetry axis. Lower part: rods and electrical connections to apply RF and DC voltages [27].

for beam accumulation, bunching, and emittance improvement $[27,41]$ of the $60 \mathrm{keV}$ ion beam delivered by ISOLDE. The ISOLDE radioactive ion beam is accumulated for a certain time (typically a few ms) so that the ions stored at the potential minimum of the trap and are ejected afterwards as a short ion bunch. Figure 3 shows the cooling of a $\mathrm{Cs}^{+}$ensemble simulated with the assumption of a viscous damping force in helium buffer gas where this model is a good approximation. However, for the understanding of the ion loss mechanisms and the final ion temperature a microscopic individual-collision model is necessary, which incorporates realistic potentials between the ions and their neutral collision partners [39].

\subsection{Ion motion in a Penning trap}

An ideal Penning trap is a superposition of a homogeneous magnetic field $\boldsymbol{B}=B \hat{z}$ with an electrostatic quadrupole potential $[31,32]$

$$
U(\rho, z) \propto 2 z^{2}-\rho^{2},
$$

with $\rho^{2}=x^{2}+y^{2}$. This allows to trap charged particles in a small, well-defined volume. A detailed derivation of the equations of motion for a single ion stored in a Penning trap is given in [37].

The electrostatic potential can be achieved by the electrode configuration as shown in fig. 4, where the trap is formed by two endcap electrodes and a ring electrode, all being hyperboloids of revolution. The quadrupole potential can as well be created by cylindrical electrodes (the ring, the endcaps, and some compensation electrodes, see fig. 4). With appropriate potentials applied to these electrodes the same quadrupole potential as in the case of an ideal trap configuration can be realized [37]. 

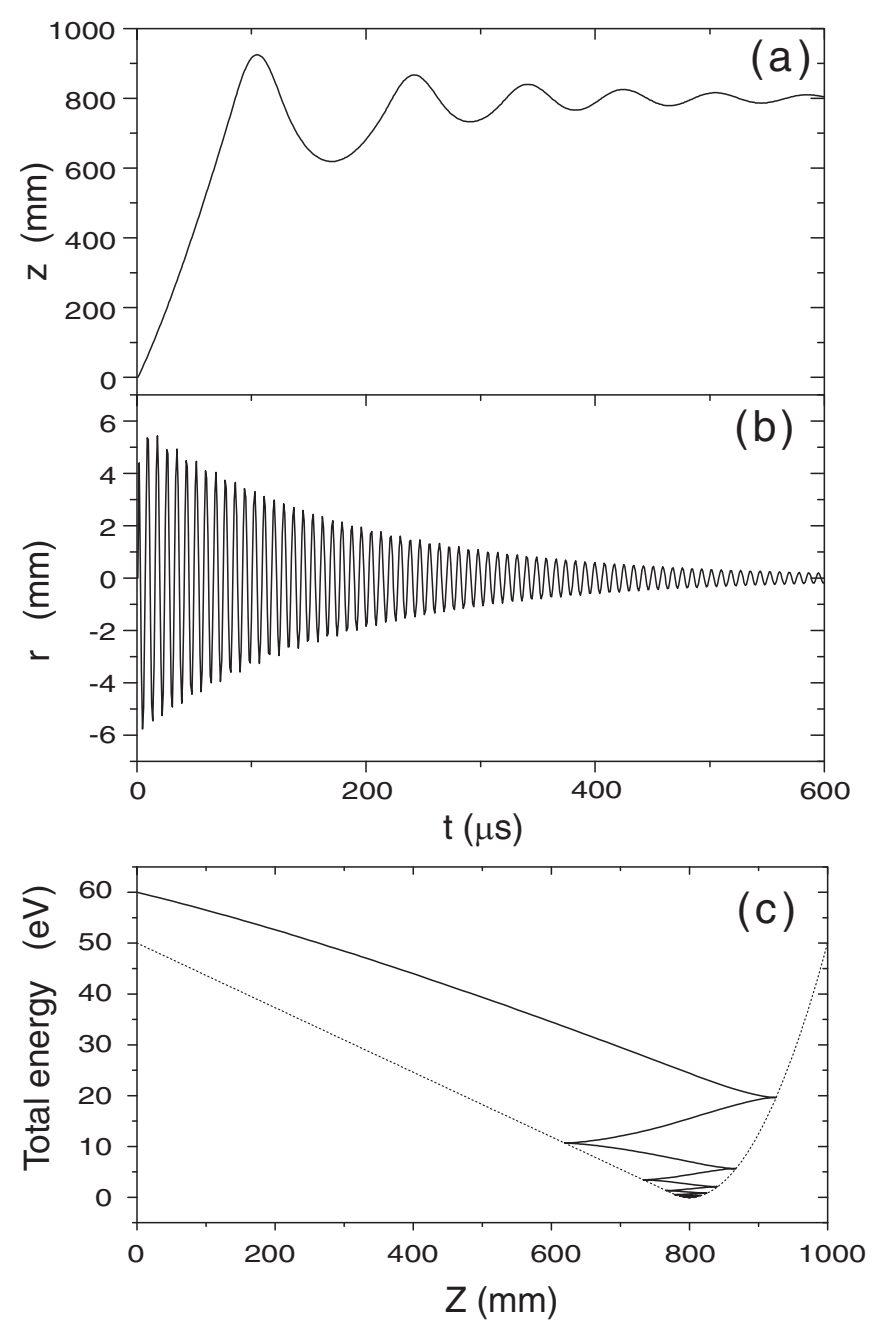

Fig. 3. Simulation of the cooling and accumulation processes in the linear radiofrequency quadrupole trap of ISOLTRAP for $\mathrm{Cs}^{+}$ions at a helium pressure of $10^{-2}$ mbar: (a) the axial position as a function of time; (b) the radial position as a function of time; (c) the total axial energy with respect to the axial position (the axial potential is also plotted) [27].

A voltage difference of $U_{0}$ applied between the endcaps and the ring electrode produces a quadrupolar potential

$$
U(\rho, z)=\frac{U_{0}}{4 d^{2}}\left(2 z^{2}-\rho^{2}\right) .
$$

The characteristic trap dimension $d$ for a Penning trap with hyperbolical electrodes is defined as

$$
4 d^{2}=\left(2 z_{0}^{2}+\rho_{0}^{2}\right),
$$

where $\rho_{0}$ denotes the inner ring radius and $2 z_{0}$ is the closest distance between the two endcaps (see fig. 4).

In an ideal electric- and magnetic-field configuration the axial motion of the ion with mass $m$ is decoupled from the magnetic field and the ion performs an harmonicoscillatory motion with the frequency

$$
\omega_{z}=\sqrt{\frac{q U_{0}}{m d^{2}}},
$$

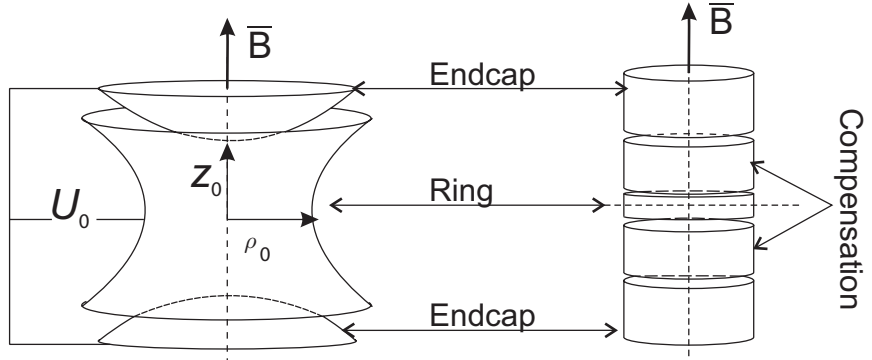

Fig. 4. Left: electrode configuration of a Penning trap consisting of two endcap electrodes and a ring electrode with hyperboloidal shape. Right: acylindrically shaped Penning trap with correction electrodes. A harmonic potential is created around the center of the ring electrode whereas the extended potential well is used for the efficient capture of the injected ion bunch [42].

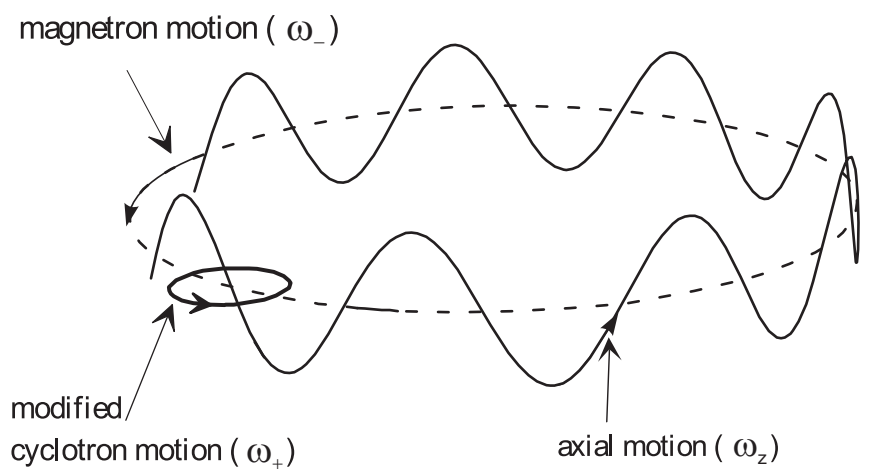

Fig. 5. The three ideally independent eigenmotions of an ion in a Penning trap: a harmonic oscillation in the axial direction (axial motion with frequency $\omega_{z}$ ), and a radial motion that is a superposition of the modified cyclotron motion with frequency $\omega_{+}$and the magnetron motion with frequency $\omega_{-}$.

where $U_{0}$ is the voltage applied between ring electrode and endcaps.

The two radial motions are known as the cyclotron motion with modified cyclotron frequency (due to the presence of the quadrupole electric field) $\omega_{+}$and the magnetron motion with eigenfrequency $\omega_{-}$:

$$
\omega_{ \pm}=\frac{\omega_{c}}{2} \pm \sqrt{\frac{\omega_{c}^{2}}{4}-\frac{\omega_{z}^{2}}{2}},
$$

with

$$
\omega_{c}=\frac{q}{m} B
$$

For typical trapping parameters the magnetron motion is a slow drift motion caused by the $\boldsymbol{E} \times \boldsymbol{B}$ field. The three eigenmotions of an ion confined in a Penning trap are shown in fig. 5. The two radial frequencies obey the relation

$$
\omega_{c}=\omega_{+}+\omega_{-} .
$$

Though there are other invariant relations, this one is the most important equation in the context of the ISOLTRAP mass spectrometer, since a direct determination of this sum frequency allows to determine the mass of the ion of 

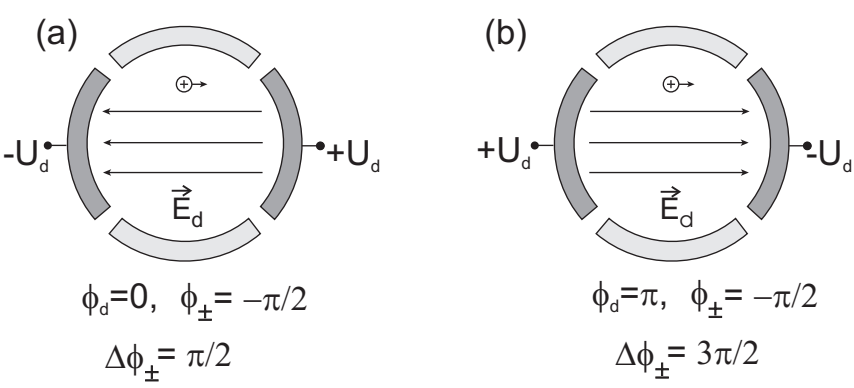

Fig. 6. (a) Electric dipolar-excitation field $\boldsymbol{E}_{\mathbf{d}}$ for $\phi_{\mathrm{d}}=0$. $\boldsymbol{E}_{\mathrm{d}}$ points in the negative $x$-direction. A positive ion at $\phi_{ \pm}=$ $-\pi / 2$ is also shown with the arrow indicating its direction of rotation. The phase difference is $\Delta \phi_{ \pm}=\pi / 2$. (b) For $\phi_{\mathrm{d}}=\pi$, $\boldsymbol{E}_{\mathbf{d}}$ points in the positive $x$-direction. The phase difference is $\Delta \phi_{ \pm}=3 \pi / 2[26]$.

interest as long as the magnetic-field amplitude $B$ and the charge state $q$ are known.

\subsection{Excitation of the ion motion in a Penning trap}

To measure the cyclotron frequency of a stored ion, to remove unwanted ion species from the trap, or to perform buffer gas cooling in a Penning trap, it is necessary to drive the ion motion with an external oscillating electric field. The effect on the ion motion depends on the multipolarity, the amplitude and the frequency of the driving radiofrequency field. At ISOLTRAP, dipolar and quadrupolar driving fields in the radial plane are applied. For this purpose the ring electrode is four-fold segmented as indicated in fig. 6 .

\subsubsection{Dipolar excitation}

Figure 6 depicts how the radiofrequency voltage is applied to the ring segments in order to achieve the dipolar excitation of the motion of the trapped ions. An oscillating dipolar potential is expressed as

$$
\tilde{U}_{\mathrm{d}}=\alpha \frac{U_{\mathrm{d}}}{\rho_{0}} \cos \left(\omega_{\mathrm{d}} t-\phi_{\mathrm{d}}\right) \cdot x
$$

where $\alpha$ is a geometric factor taking into account the influence of the endcaps on the potential and that in the azimuthal plane, the shape of the ring electrode segment does not follow the dipolar equipotential lines. The applied dipolar field has an amplitude $U_{\mathrm{d}}$, frequency $\omega_{\mathrm{d}}$ and phase $\phi_{\mathrm{d}}$, respectively. The phase of the ion motion is denoted by $\phi_{ \pm}$. A dipolar excitation applied at any of the three eigenfrequencies can excite that motion without affecting the others. These kinds of excitations at $\omega_{-}$and $\omega_{+}$frequencies are in use to enlarge the magnetron and cyclotron radii and to remove unwanted species from the trap.

In [26] it is shown that in case the radius of the resonantly excited initial ion motion is greater than zero, the phase difference between the ion eigenmotion and the dipolar excitation signal plays an important role on the evolution of the ion radius due to the excitation. The time evolution of the cyclotron and the magnetron radii are affected oppositely and depend on the phase difference $\Delta \phi_{ \pm}$. Three scenarios of the phase differences are discussed here:

$-\Delta \phi_{ \pm}=\pi / 2$ : this situation is illustrated in fig. 6(a), where the dipolar excitation field $E_{\mathrm{d}}$ is antiparallel to the ion motion. In the case of resonant cyclotron excitation, $\rho_{+}(t)$ decreases linearly with $t$ down to zero. Then it grows linearly with $t$. In the case of resonant magnetron excitation, $\rho_{-}(t)$ grows linearly with $t$ from the very beginning of the excitation (see fig. $7(\mathrm{a})$ ).

$-\Delta \phi_{ \pm}=3 \pi / 2$ : this situation is illustrated in fig. $6(\mathrm{~b})$, where the dipolar excitation field $E_{\mathrm{d}}$ is parallel to the ion's motion. In the case of resonant reduced-cyclotron excitation, $\rho_{+}(t)$ grows linearly with $t$ from the very beginning of the excitation. In the case of resonant magnetron excitation, $\rho_{-}(t)$ diminishes linearly with $t$ to zero and then it grows linearly with $t$ (see fig. $7(\mathrm{a})$ ).

$-\Delta \phi_{ \pm}=0$ : in this case the radius grows slowly at the beginning, then almost linearly with the excitation time (see fig. $7(\mathrm{a})$ ).

In fig. $7(\mathrm{~b})$, the magnetron radius $\rho_{-}(t)$ is plotted as a function of the phase difference $\Delta \phi_{-}$for three different excitation times: $t=1 / \nu_{-}$(one magnetron period), $t=$ $2 A_{0} / k_{0}$ (a bit more than two periods) where the amplitude becomes zero for $\Delta \phi_{-}=\pi / 2$ (see fig. $7(\mathrm{a})$ ), and $t=10 / \nu_{-}$ (ten magnetron periods). Considering the excitation time applied at the ISOLTRAP experiment the last case is the one which is of highest interest since at a phase difference of $\pi / 2$ between the ion's magnetron phase and the applied $\mathrm{RF}$ excitation the fastest increase in the magnetron radius is obtained. This scheme is known as magnetron phaselocking [26].

\subsubsection{Quadrupolar excitation}

An oscillating quadrupolar potential has the form

$$
\tilde{U}_{\mathrm{q}}=\alpha \frac{U_{\mathrm{q}}}{\rho_{0}^{2}} \cos \left(\omega_{\mathrm{q}} t-\phi_{\mathrm{q}}\right) \cdot\left(x^{2}-y^{2}\right),
$$

where $\alpha$ is again a geometric factor. The quadrupolar field due to this potential is given by

$$
\boldsymbol{E}_{\mathbf{q}}=-\nabla \tilde{U}_{\mathrm{q}}=-2 \alpha \frac{U_{\mathrm{q}}}{\rho_{0}^{2}} \cos \left(\omega_{\mathrm{q}} t-\phi_{\mathrm{q}}\right) \cdot\left[\begin{array}{r}
x \\
-y \\
0
\end{array}\right] .
$$

In case the frequency of the exciting RF field is exactly set to $\omega_{c}$, the radial amplitude evolves as

$$
\begin{array}{r}
\rho_{ \pm}(t)=\rho_{ \pm}(0) \cos \left(\frac{\omega_{\text {conv }}}{2} t\right) \mp \\
\rho_{\mp}(0) \sin \left(\frac{\omega_{\text {conv }}}{2} t\right) \cos \left(\phi_{\mathrm{q}}-\phi_{+}-\phi_{-}\right),
\end{array}
$$

where

$$
\omega_{\mathrm{conv}}=\frac{k_{0}}{2\left(\omega_{+}-\omega_{-}\right)},
$$



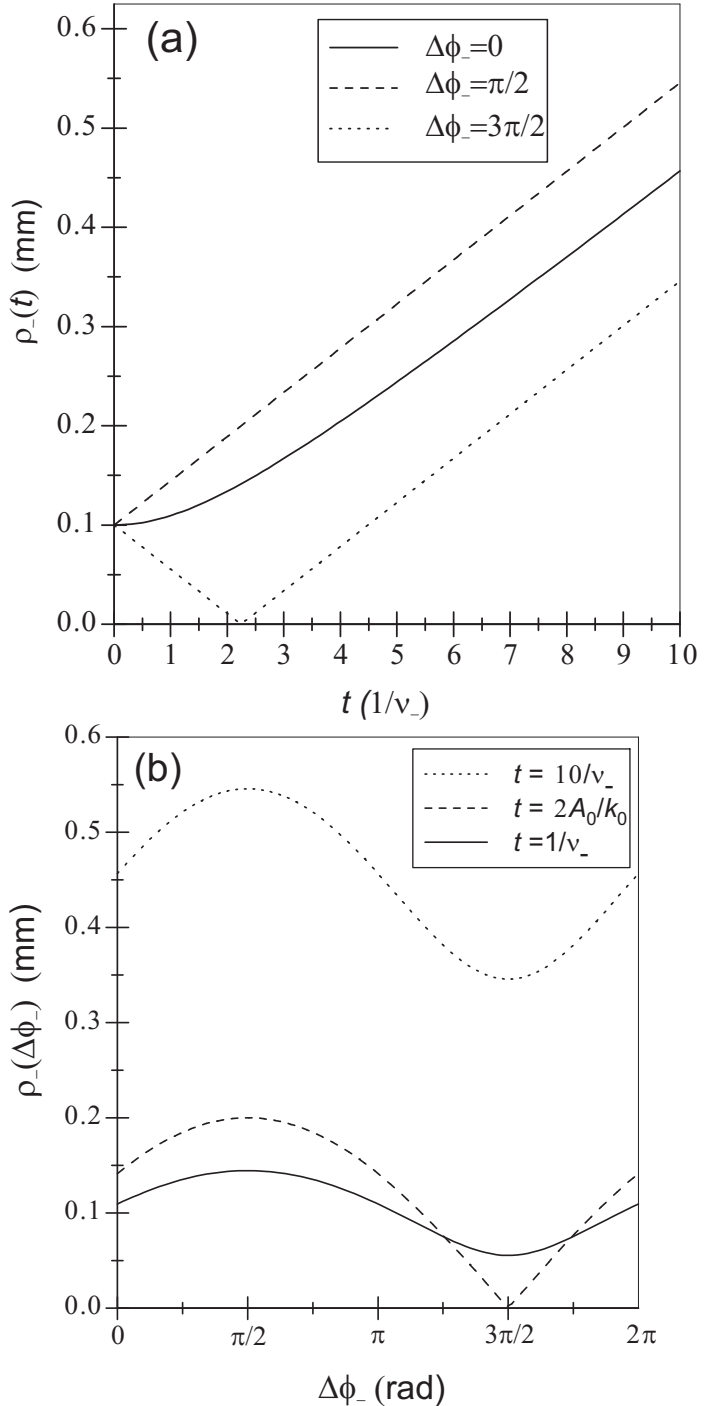

Fig. 7. (a) Dipolar excitation of the magnetron motion in a Penning trap. The magnetron radius is plotted versus the excitation time (given in units of the magnetron period) for three phase differences $\Delta \phi_{-}=0, \pi / 2$, and $3 \pi / 2$. For $\Delta \phi_{-}=0$ (solid curve) it grows slowly at first, then almost linearly, for $\Delta \phi_{-}=$ $\pi / 2$ (dashed line) it grows linearly from the very beginning of the excitation and for $\Delta \phi_{-}=3 \pi / 2$ (dotted line) it first shrinks to zero before it grows linearly with $t$. (b) Magnetron radius plotted versus $\Delta \phi_{-}$for three different excitation times $t: t=1 / \nu_{-}$(solid line), $t=2 A_{0} / k_{0}$ (dashed line) where the amplitude becomes zero for $\Delta \phi_{-}=3 \pi / 2$, and $t=10 \nu_{-}$(dotted line) [26].

and $k_{0}$ is given by

$$
k_{0}=\alpha \frac{q}{m} \frac{U_{\mathrm{q}}}{\rho_{0}} .
$$

This type of excitation is also known as sideband excitation. In case the phase difference between the excitation and the radial motion $\phi_{\mathrm{q}}-\phi_{-}-\phi_{+}$is equal to $\pi$, the radial evolution is simplified to

$$
\rho_{ \pm}(t)=\rho_{ \pm}(0) \cos \left(\frac{\omega_{\text {conv }}}{2} t\right) \pm \rho_{\mp}(0) \sin \left(\frac{\omega_{\text {conv }}}{2} t\right) .
$$
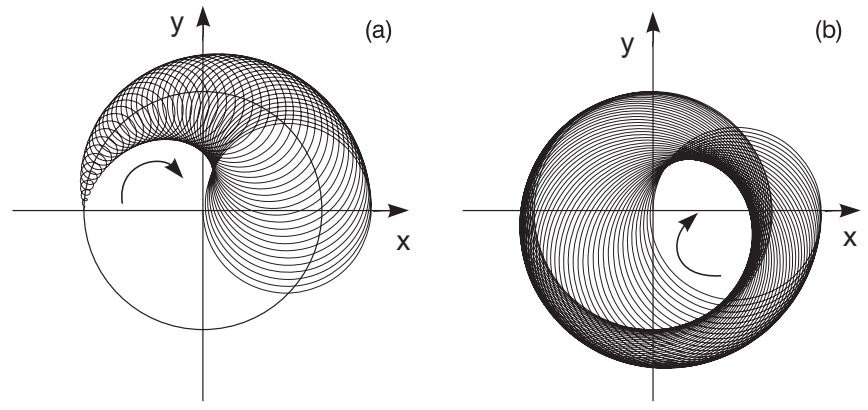

Fig. 8. Radial ion motion with a quadrupolar excitation at $\omega_{\mathrm{q}}=\omega_{c}$ in the plane perpendicular to the magnetic field. The motion starts with pure magnetron motion (a), indicated by the circle, and converts fully to cyclotron motion (b) in the time interval $T_{\text {conv }}$.

There is a continuous conversion with frequency $\omega_{\text {conv }}$ between the two radial motions. A complete conversion from one motion to the other therefore takes $T_{\text {conv }}=\pi / \omega_{\text {conv }}$. Figure 8 shows this conversion as a function of time. For a mass of $A=39$, a conversion time of $\approx 100 \mathrm{~ms}$ is obtained for an rf-amplitude of about $10 \mathrm{mV}$ applied to the radial segments of the ring electrode at ISOLTRAP's precision trap.

\subsection{3 lon cooling and mass-selective centering}

In a gas-filled Penning trap the ions lose their kinetic energy due to collisions with the buffer gas atoms (see sect. 2.1). Solving the equations of motion including the viscous force, one obtains the time evolution of the ion's coordinates as

$$
\begin{aligned}
{\left[\begin{array}{l}
x \\
y
\end{array}\right]=} & \rho(0)_{-} e^{-\alpha_{-} t}\left[\begin{array}{r}
\cos \left(\omega_{-}^{\prime} t-\phi_{-}\right) \\
-\sin \left(\omega_{-}^{\prime} t-\phi_{-}\right)
\end{array}\right] \\
& +\rho(0)_{+} e^{-\alpha_{+} t}\left[\begin{array}{c}
\cos \left(\omega_{+}^{\prime} t-\phi_{+}\right) \\
-\sin \left(\omega_{+}^{\prime} t-\phi_{+}\right)
\end{array}\right],
\end{aligned}
$$

where

$$
\omega_{ \pm}^{\prime}=\omega_{ \pm} \pm \Delta \omega, \quad \Delta \omega=\frac{1}{16} \cdot\left(\frac{\delta}{m}\right)^{2} \cdot \frac{8 \omega_{z}^{2}+\left(\frac{\delta}{m}\right)^{2}}{\left(\omega_{c}^{2}-2 \omega_{z}^{2}\right)^{\frac{3}{2}}} .
$$

Here some of the higher-order terms have been neglected. The radial motion in the presence of a damping force is still composed of two circular motions. However, compared to the undamped case, the frequencies are now shifted up and down, respectively, by $\Delta \omega$ from the reduced cyclotron $\omega_{+}$and the magnetron $\omega_{-}$frequencies. These frequency shifts are very small and can be neglected in the case of ISOLTRAP. In addition, since the shifts are equal in magnitude but opposite in direction, their sum and hence $\omega_{c}$ remains unchanged.

Neglecting some of the higher-order terms, the radii of the motional modes change exponentially with inverse the time constants $\alpha_{ \pm}$given by,

$$
\alpha_{ \pm}=\frac{\delta}{2 m}\left\{1 \pm\left(1+\frac{1}{8} \cdot \frac{8 \omega_{z}^{2}+\left(\frac{\delta}{m}\right)^{2}}{\omega_{c}^{2}-2 \omega_{z}^{2}}\right)\right\} .
$$



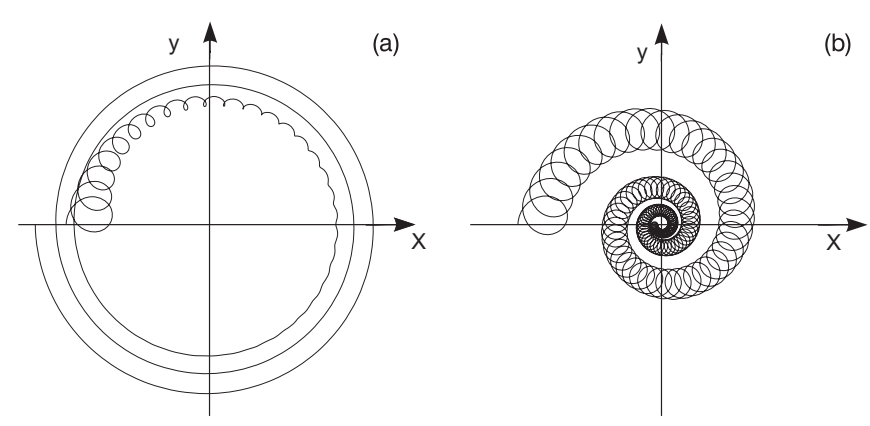

Fig. 9. Radial motion of an ion in a buffer gas-filled Penning trap in the plane perpendicular to the magnetic field. The crossing of the $x$ and $y$ coordinates marks the center of the trap. A velocity-dependent damping force representing the buffer gas cooling has been included. In (a) a fast damping of the cyclotron motion and a slow increase of the magnetron motion are observed. In (b) the effect of an additional excitation with an azimuthal quadrupolar field of frequency $\nu_{c}$ is shown. Both the cyclotron and the magnetron motion are decreased and a mass-selective centering to the trap center is achieved.

While the radius of the modified cyclotron motion decreases with an inverse time constant $\alpha_{+} \approx \delta / m$, the magnetron radius increases with a much smaller inverse time constant $\alpha_{-} \approx-(\delta / 2 m) \cdot\left(\omega_{z} / \omega_{c}\right)^{2}$ as shown in fig. 9(a). Due to its low frequency, the magnetron motion has mainly potential (negative) energy. Hence, cooling by buffer gas collisions increases the radius of the magnetron orbit, finally leading to a loss of the ions. By applying a quadrupolar rf-field at $\omega_{c}$, the magnetron motion is coupled to the cyclotron motion. Therefore one observes a slow increase in the magnetron radius as compared to the fast decrease of the cyclotron radius. Without quadrupolar excitation at $\omega_{c}$, the particle will be lost due to the increase of the magnetron radius. This can be avoided by coupling the two radial motions. Hence, both radii will decrease as shown in fig. 9(b). This technique is known as mass-selective buffer gas centering [25].

\subsection{Time-of-flight detection of the cyclotron resonance}

The principle of the time-of-flight (TOF) detection of the cyclotron resonance was originally proposed by Bloch [43] and first applied for mass measurements by Gräff et al. [44]. Though it is a destructive detection method, its advantage is obvious: It can be used for a single or a low number of stored ions in the trap and is applicable to all ions and avoids, for example, tuning of resonance circuits. This is of particular advantage for very short-lived radioactive ions where the nuclear decay anyhow limits the storage and observation time in the trap.

Ions captured and stored in the center of the trap are first excited by an azimuthal dipolar excitation at their magnetron frequency, which leads to a magnetron radius $\rho_{-0}$. An azimuthal quadrupolar rf-excitation is then applied for a time period of $T_{\mathrm{q}}=\pi / \omega_{\text {conv }}$. In the case of $\omega_{\mathrm{q}}=\omega_{c}$, the magnetron motion is fully converted to cyclotron motion as discussed in sect. 2.4.2. Finally, the cy-
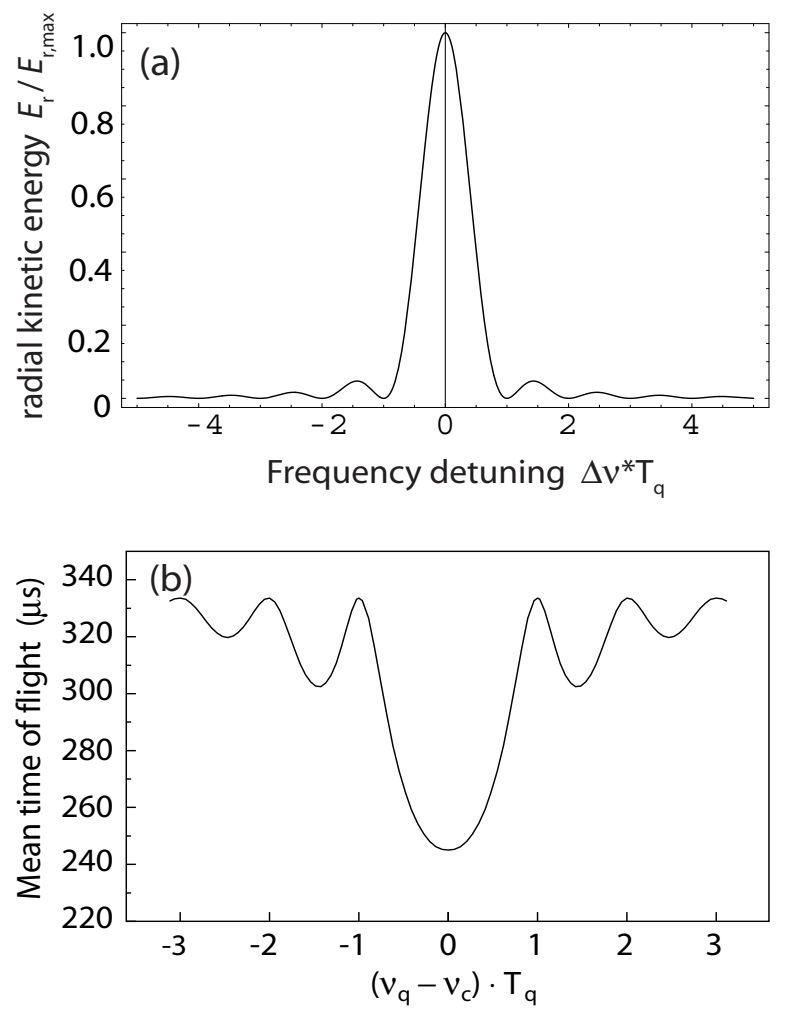

Fig. 10. (a) Gain in radial energy of the ion motion in the case of quadrupolar excitation near $\nu_{c}$ as a function of the detuning $\left(\nu_{\mathrm{q}}-\nu_{c}\right)$ as given in eq. (21). (b) The theoretical line shape of the mean time of flight in the case of quadrupolar excitation near $\nu_{c}$ as a function of the detuning $\left(\nu_{\mathrm{q}}-\nu_{c}\right)$. The central frequency is the true cyclotron frequency.

clotron radius becomes equal to the initial magnetron radius. Since the reduced cyclotron frequency is much higher than the magnetron frequency, this conversion is accompanied by an increase in the radial kinetic energy

$$
E_{r}(t) \propto \omega_{+}^{2} \rho_{+}(t)^{2}-\omega_{-}^{2} \rho_{-}(t)^{2} \approx \omega_{+}^{2} \rho_{+}(t)^{2} .
$$

Off-resonance $\left(\omega_{\mathrm{q}} \neq \omega_{c}\right)$ the conversion is not complete and hence the radial energy gain smaller. The exact functional form of the energy gain depends on the envelope of the excitation signal. For a rectangular envelope, i.e., a quadrupolar excitation switched on with constants amplitude for a certain time interval $T_{\mathrm{q}}$, the final radial kinetic energy is of the form [45] (see fig. 10(a)):

$$
E_{r} \propto \frac{\sin ^{2}\left(\omega_{\mathrm{b}} T_{\mathrm{q}}\right)}{\omega_{\mathrm{b}}^{2}},
$$

with

$$
\omega_{\mathrm{b}}=\frac{1}{2} \sqrt{\left(\omega_{\mathrm{q}}-\omega_{c}\right)^{2}+\left(\omega_{\mathrm{conv}} / 2\right)^{2}} .
$$

After this sequence of excitations, the ions are axially released as an ion pulse from the trap through a drift tube and their individual time of flight is recorded by a detector installed about $1.2 \mathrm{~m}$ upstream. The MCP or channeltron is installed where the magnetic-field amplitude is already reduced by three orders of magnitude. Thus, the ions cross 
the region of high magnetic-field gradient. The magnetic moment

$$
\mu=E_{r}\left(\omega_{\mathrm{q}}\right) / B
$$

that the ions acquire due to their radial motion, interacts with the magnetic-field gradient and hence the ions experience an axial force

$$
\boldsymbol{F}=-\boldsymbol{\mu}(\boldsymbol{\nabla} \cdot \boldsymbol{B})=-\frac{E_{r}}{B} \frac{\partial B}{\partial z} \hat{z} .
$$

This force leads to a reduction in the time of flight from the trap center $(z=0)$ to the detector $\left(z=z_{1}\right)$. This time of flight for a given radial energy $E_{r}$ can be calculated by

$$
T_{\text {tot }}\left(\omega_{\mathrm{q}}\right)=\int_{0}^{z_{1}} \sqrt{\frac{m}{2\left(E_{0}-q U(z)-\mu\left(\omega_{\mathrm{q}}\right) B(z)\right)}} \mathrm{d} z,
$$

where $E_{0}$ is the initial axial kinetic energy of the ion, $U(z)$ is the electrostatic potential along the flight path and $B(z)$ is the magnetic field. A scan of the quadrupolar excitation frequency $\omega_{\mathrm{q}}$ around $\omega_{c}$ thus produces a characteristic time-of-flight resonance [45] (see fig. 10(b)).

It follows from eqs. (20), (21), and (25) that the depth of the main peak is a function of the initial magnetron radius $\rho_{0}$. The depth is a measure for the radial energy gain of the ions: A higher gain leads to a greater acceleration after ejection, a shorter TOF, and consequently an enhanced resonance TOF effect. The completeness of the conversion from pure magnetron to pure cyclotron motion depends on the amplitude $U_{\mathrm{q}}$ and on the duration $T_{\mathrm{q}}$ of the excitation. The line width of the centroid is inversely proportional to $T_{\mathrm{q}}$. Thus, the resolving power $R \approx \nu_{\mathrm{q}} \cdot T_{\mathrm{q}}$ depends on the excitation time.

\subsection{Real Penning trap}

A real Penning trap deviates from an ideal one in many respects. Trap imperfections lead to shifts in the eigenfrequencies and hence to systematic uncertainties in the mass determination [37]. Knowledge about these imperfections and their influence is therefore essential for the design of a Penning trap mass spectrometer and to understand the possible systematic uncertainties in mass determination. The most important trap imperfections will be briefly described in the following.

The electric field in a real Penning trap may deviate from a pure quadrupole one. Only the lowest two multipole orders, namely, the octupole and the dodecapole contributions, become significant as compared to the required quadrupole contribution for most practical purposes. They lead to a mass-dependent frequency shift and therefore a high-precision mass measurement requires the mass calibration to be performed with an ion close in mass to that of the ion under investigation [24,37]. It is also possible to minimize these contributions by having a large characteristic trap dimension, small trapping potential, and small amplitude of ion motion.

Thus, it is desired to have mass references as close as possible. Mass doublets, i.e. ions with identical mass numbers $A$ are the best choices but in most cases such reference ions with well-known masses are not available. So the mass references of choice are carbon clusters $\mathrm{C}_{n}$ (with $n=1,2,, 3, \ldots$ ) $[46,47]$ (see sect. 3.2.2).

Electric-field imperfections can also lead to frequency shifts via the induced image charges of the ions on the trap electrodes. This shift scales inversely to the cube of the trap dimension and hence the effect is pronounced for a small trap [48]. Since $\Delta \omega_{+}=-\Delta \omega_{-}$no frequency shift will occur in the ISOLTRAP mass measurement procedure since it determines the sum frequency given in eq. (9).

A tilt of the electrostatic-field axis relative to the magnetic-field axis can give rise to a systematic uncertainty in mass determination as well [37]. This misalignment shifts all eigenfrequencies. For the sum frequency (9) the resulting shift $\Delta \omega_{c}^{\text {tilt }}$ can be calculated [24] and is found to be mass independent and, for $\Theta \ll 1$, to be proportional to the square of the tilting angle $\Theta$ [37]:

$$
\Delta \omega_{c}^{\mathrm{tilt}} \approx \frac{9}{4} \omega_{-} \sin ^{2} \Theta .
$$

This frequency shift contributes to a systematic uncertainty and therefore the trap has to be aligned very carefully.

Accurate mass determination demands an excellent magnetic-field homogeneity and temporal stability. Commercial superconducting magnets nowadays can provide a homogeneity of $\Delta B / B \leq 10^{-7}$ over a volume of $1 \mathrm{~cm}^{3}$. The magnetic-field homogeneity can easily be destroyed if materials with high magnetic susceptibility are introduced into the magnetic field. Such materials can be part of the trap itself. Therefore, care is taken and usually oxygen-free high-conductivity copper and glass ceramics are used as trap and insulation materials. Nevertheless, their susceptibilities are high enough to see noticeable perturbations in the magnetic field.

Frequency shifts occur if the magnetic field is a function of the even powers of the distance from the trap center [37]. A stored ion then experiences different average magnetic fields for different motional amplitudes. The lowest-order inhomogeneity of interest is a magnetic hexapole component. It creates a frequency shift given by

$$
\Delta \omega_{c}^{\operatorname{magn}} \approx \beta_{2} \omega_{c}\left(\rho_{z}^{2}-\rho_{-}^{2}\right)
$$

where $\beta_{2}$ denotes the relative strength of the hexapole component of the magnetic field. In contrast to the frequency shifts discussed above, this shift is now proportional to the cyclotron frequency of the stored ion and does not give rise to calibration errors provided the motional amplitudes are the same for both ion species. Since this can only be achieved within certain limits, it is still important to construct the trap such that the inhomogeneity is smaller than the aimed mass accuracy.

Magnetic-field instability is another point of concern and is determined by the magnet itself, ambient magneticfield changes, pressure changes in the helium cryostat, and temperature changes of the experimental equipment installed in the magnet bore [49-52]. The demand for the 
magnetic-field stability is defined by the desired mass accuracy and by the switching time between the cyclotron frequency measurements of the reference ion and the ion of interest. The available field stability is $(\delta B / B) / \delta T<$ $10^{-9} / \mathrm{h}$ at ISOLTRAP.

In the ISOLTRAP experiment the cyclotron frequency is measured for a small number of ions stored simultaneously in the trap (typically 1-5). Thus, the effect of the Coulomb interaction on the ion motion must be taken into account to obtain the proper cyclotron frequency. It has been observed and investigated for both, Paul [53] and Penning traps [54-57]. The effects in a Penning trap will be addressed here.

If the simultaneously stored ions are of the same mass, the driving frequency acts on the $q / m$ center of the stored ion cloud and no frequency shift is observed [58]. For stored ions of different mass (contaminations), a frequency shift of $\Delta \omega_{c}^{\mathrm{Cbc}}$ in the obtained resonance is observed. It was found [55] that the sign of $\Delta \omega_{c}^{\mathrm{Cbc}}$ depends strongly on the cyclotron frequency difference of the stored species compared to the line width of the resonance. The shift increases with the total number of stored ions. In case that the unperturbed resonances cannot be resolved, only a single resonance is observed, which is narrower than expected from simple superposition of the individual resonances. The average mass of all ions stored in the trap determines the position of the resonance. For large mass differences of the ion species, the measured cyclotron frequency of both species is shifted to lower frequencies. The size of the shift of one species is found to be proportional to the number of stored ions of the other species and vice versa.

The coupling of all eigenmotions by Coulomb interaction must be taken into account for a quantitative description of the observed frequency shifts. Until now no general analytical solution has been found for the equation of motion. However, it is possible to confirm the observations qualitatively by a three-dimensional simulation of the motion of simultaneously stored ions [55].

In practice, it is desirable to have a pure ion ensemble stored in the trap. Alternatively, the cyclotron frequency can be extrapolated to a single stored ion in the trap by analyzing the cyclotron frequencies for different numbers of stored ions [59] (see sect. 5.1).

\section{Experimental setup of ISOLTRAP}

The ISOLTRAP mass spectrometer started its operation in 1986 at the on-line mass separator ISOLDE-2 at CERN, Geneva [60,61]. In 1992 it was moved to its present place at the PS-Booster [28,62]. Since the last major ISOLTRAP status report in 1996 [14], there have been many changes in the setup as well as improvements in its performances.

\subsection{Production of radioactive beams at ISOLDE}

Radionuclides are produced at ISOLDE by bombarding a thick target (for example $\mathrm{UC}_{2}$ ) with up to $1.4 \mathrm{GeV}$ protons from the CERN proton synchrotron (PS) booster (up

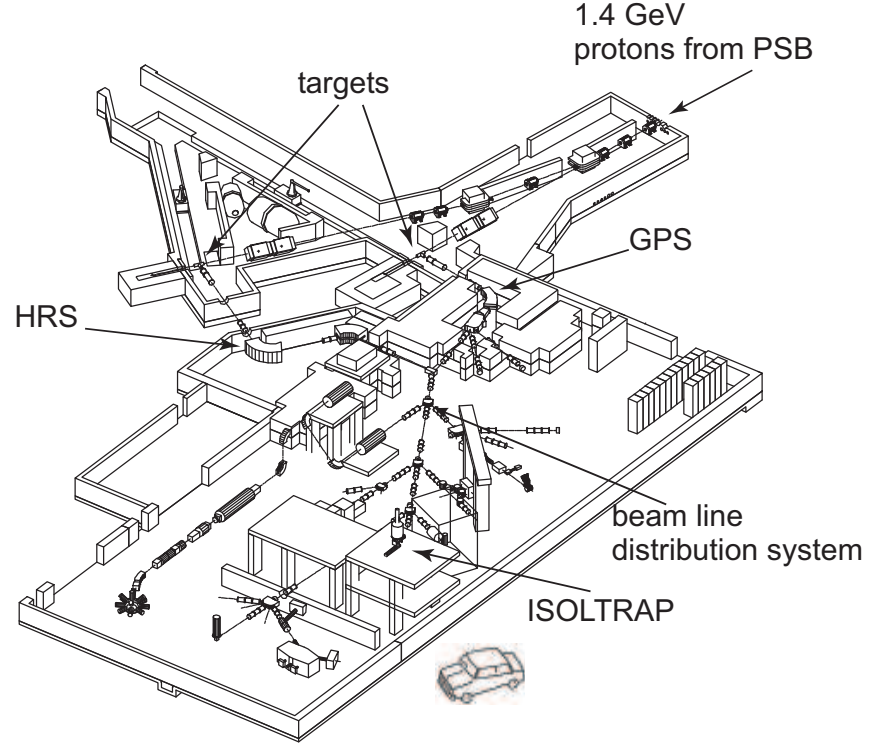

Fig. 11. The ISOLDE hall with different beam lines for beam distribution towards different experiments (sketch of the car for scale).

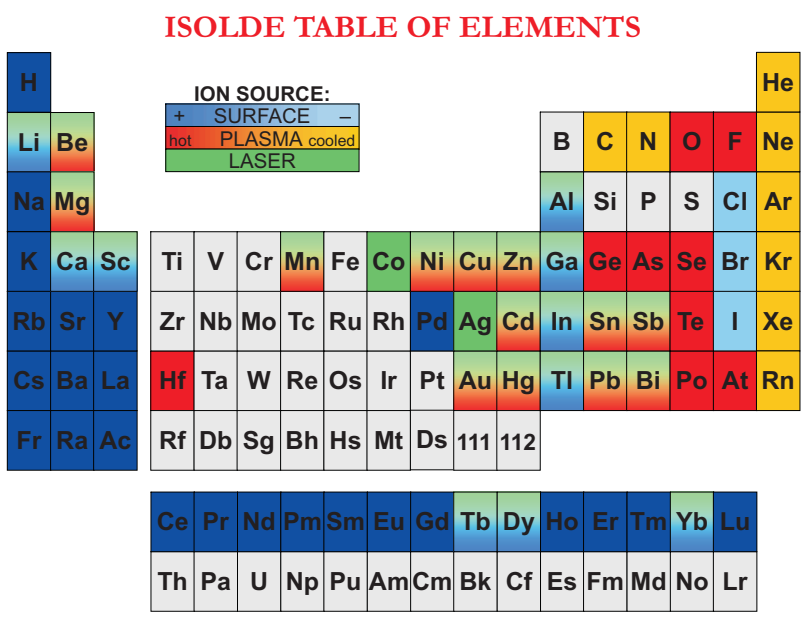

Fig. 12. (Color online) Color-coded periodic table of elements and the ion sources in use for the on-line production of shortlived nuclides at ISOLDE/CERN. (Courtesy of L. Fraile.)

to $3 \times 10^{13}$ protons/pulse) [28]. Presently close to 1100 radionuclides of more than 70 elements are available. The layout of the ISOLDE experimental hall is shown in fig. 11. There are two target stations and two mass separator units, the General Purpose Separator (GPS) with a typical mass-resolving power of $R \sim 1000$ and the High Resolution Separator (HRS) with $R$ up to 5000 [28]. Radioactive products from fission, fragmentation or spallation reactions diffuse out of the target. In fig. 12 the commonly used ionization techniques for the ion production are indicated for the different elements. Prior to the advent of the Resonance Ionization Laser Ion Source (RILIS), most often surface ionization is employed. Alternatively, plasma or 


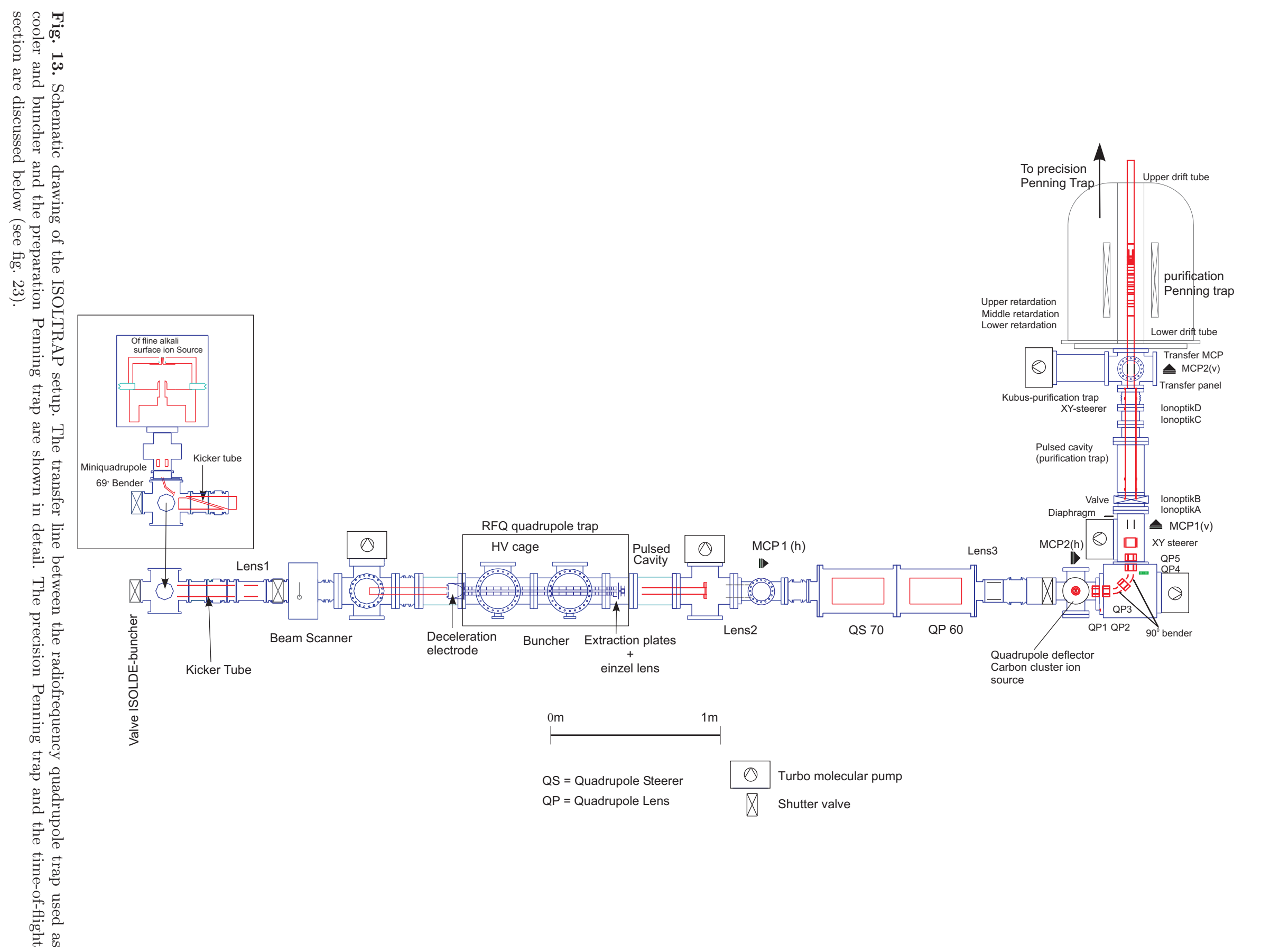


laser ionization are available. The laser ion source provides the strongest suppression of isobaric contaminations [63].

After ionization in the ion source the ions are accelerated to $60 \mathrm{keV}$, mass separated by magnetic sector fields and delivered via transfer beam lines to different experimental setups. The ion beam produced in this way is transported about $30 \mathrm{~m}$ downstream to the ISOLTRAP setup, installed at the central beamline of ISOLDE. For the guidance of the ion beam lenses and deflectors are used. Figure 13 shows a technical layout of the ISOLTRAP beamline whose main parts will be discussed in detail in the following sections.

\subsection{Off-line ion sources}

ISOLTRAP uses two different types of reference ion sources for its off-line mass and test measurements as well as for magnetic-field calibrations during on-line mass measurements of radioactive ions: a surface ion source mainly delivering ions of alkali elements and a laser desorption source for carbon cluster ions used for the study of the mass uncertainty limit of ISOLTRAP $[46,59,64]$.

\subsubsection{Surface ion source for alkali ions}

Up to recently the reference ion source for alkali ions used a heated tungsten ionizer for the evaporation and ionization of ${ }^{23} \mathrm{Na},{ }^{39,41} \mathrm{~K},{ }^{85,87} \mathrm{Rb}$, and ${ }^{133} \mathrm{Cs}$ atoms. All masses are known with a relative uncertainty of $10^{-9}$ or better [1]. The wanted alkali element is confined in zeolite by ion exchange and released by heating the zeolite typically to $1000^{\circ} \mathrm{C}$ depending on their vapor pressure. Tungsten wires are used within the oven to increase the ionization efficiency.

In the meantime a directly heated graphite furnace and ionizer (see fig. 14) has replaced the alkali ion source. In addition to a surface ionizer it includes a cross-beam electron impact ion source where atoms or molecules from a gas inlet or released by the furnace are ionized. A radiofrequency quadrupole mass filter is placed behind the ionizer for mass separation with a resolving power of about 200-500. The ions are finally extracted via a conical extraction electrode. This ion source is placed in front of the RFQ cooler and buncher, perpendicular to the ISOLDE beam line (see fig. 13) in order to allow a full off-line test of the whole ISOLTRAP mass spectrometer. Ions created in this way are injected into the RFQ cooler and buncher by applying proper voltages to a kicker and bender electrode. In order to create the ions at an energy close to that of the ISOLDE beam, the ion source is placed at a high-voltage platform of $60 \mathrm{kV}$. Similar ion optical conditions for both the off-line ion source and the ISOLDE beam allow fast switching between stable reference and radioactive ion beams. This in turn minimizes the systematic uncertainty due to the temporal drifts of the magnetic field.

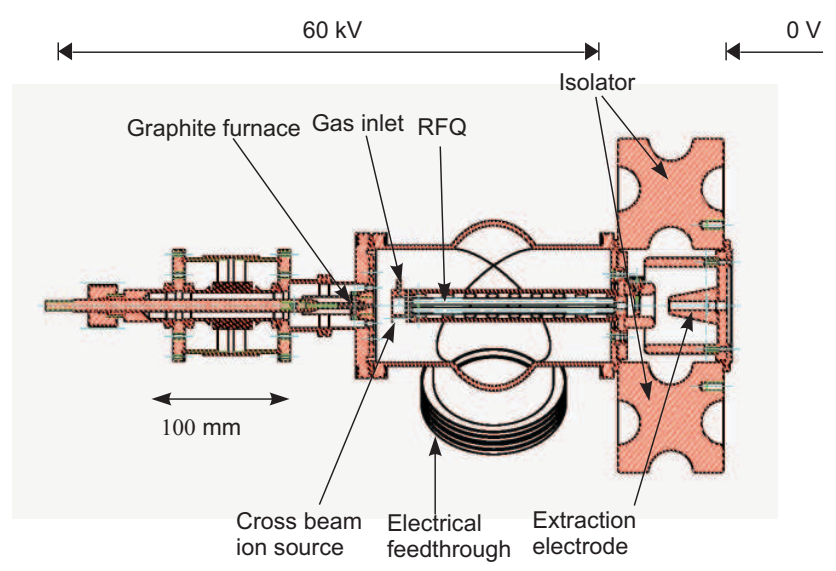

Fig. 14. Design of the new combined surface and cross-beam ion source together with a quadrupole mass filter.

\subsubsection{Ion source for carbon clusters}

Cluster ions are ideally suited for the calibration of mass spectrometers and for consistency checks and studies of systematic uncertainties in high-precision mass determination $[46,65,66]$ (see also sect. 2.6). In this context, carbon clusters provide the reference mass of choice: A multitude of reference masses all over the nuclear chart are available which are at most six atomic mass units away from any nuclide of interest. Thus, any systematic massdependent uncertainty, which increases with the difference between the measured and the reference mass, is minimized. Furthermore, the use of carbon clusters as mass references allows to perform not only direct but also absolute mass measurements since the building blocks of carbon clusters ${ }^{12} \mathrm{C}_{n}$ are related as closely as possible to the microscopic mass standard: The unified atomic mass unit $\mathrm{u}$ is by definition $1 / 12$ of the mass of ${ }^{12} \mathrm{C}$.

For the study of the systematic uncertainties of ISOLTRAP, a laser ion source was used to desorb, fragment, and ionize $\mathrm{C}_{60}$ fullerenes [59,64]. Figure 15(A) shows a typical time-of-flight spectrum of the clusters as created from a fullerene source. The carbon cluster source was mounted two meters below the preparation Penning trap, and the $90^{\circ}$ electrostatic bender between quadrupoles QP1 and QP3 was removed. Recently, a new carbon cluster laser ion source has been developed (see fig. 16) for absolute mass measurements during on-line runs. This source is mounted at a position just in front of the quadrupole QP1 (see fig. 13), which allows a fast switching between radioactive ions and carbon cluster ions since the $90^{\circ}$ bender stays in place. The ions are created at a potential of $2.5 \mathrm{kV}$, so their kinetic energy corresponds to that of the ions coming from the RFQ buncher. The source consists of a sample holder, an extraction electrode with a small aperture, and two sets of einzel lenses to focus the beam at the center of a four-rod quadrupole deflector situated at the place of the movable MCP2(h) (see fig. 13). This will ensure efficient capture of the ions in the preparation Penning trap of ISOLTRAP. 

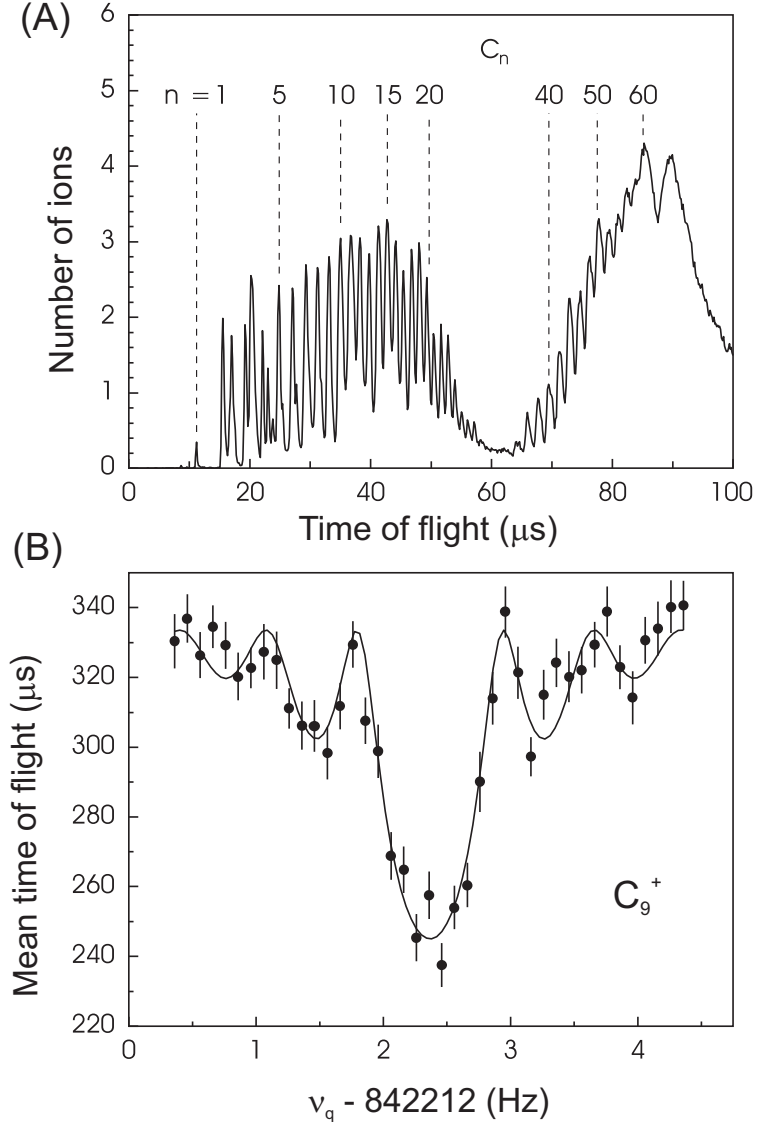

Fig. 15. Typical time-of-flight mass spectrum (A) and a timeof-flight cyclotron resonance plot for $\mathrm{C}_{9}^{+}$(B) measured with fullerene fragments produced from a carbon cluster ion source.

If quick exchange of the sample is required, one can close the shutter valve and vent the lower part without affecting the rest of the vacuum system. The sample holder is placed on a rotatable feedthrough and the laser spot is focussed off center to a spot size of 100-200 $\mu \mathrm{m}$, thereby avoiding to hit the same spot all the time. This ensures longevity of the sample. This kind of arrangement allows, in addition, to have different samples at the same time on the sample holder. The laser repetition rate as well as the intensity can be adjusted with respect to the sample of interest, the ion bunch intensity, and the measurement cycle. The angle of inclination of the laser beam with respect to the sample surface is $52^{\circ}$. The laser in use is a frequencydoubled Nd-YAG nanosecond pulsed laser $(\lambda=532 \mathrm{~nm})$ with an average power of $P_{\mathrm{av}}=15 \mathrm{~mW}$. The measured energy distribution of the produced ions is about $20 \mathrm{eV}$ at a beam energy of $2.5-3 \mathrm{keV}$, which matches well with the requirements for efficient trap loading.

\subsection{RFQ cooler and buncher}

A linear radiofrequency quadrupole (RFQ) cooler and buncher (fig. 17) has been developed at ISOLTRAP for beam emittance improvement and bunching of the quasicontinuous ISOLDE ion beam. It has been described in

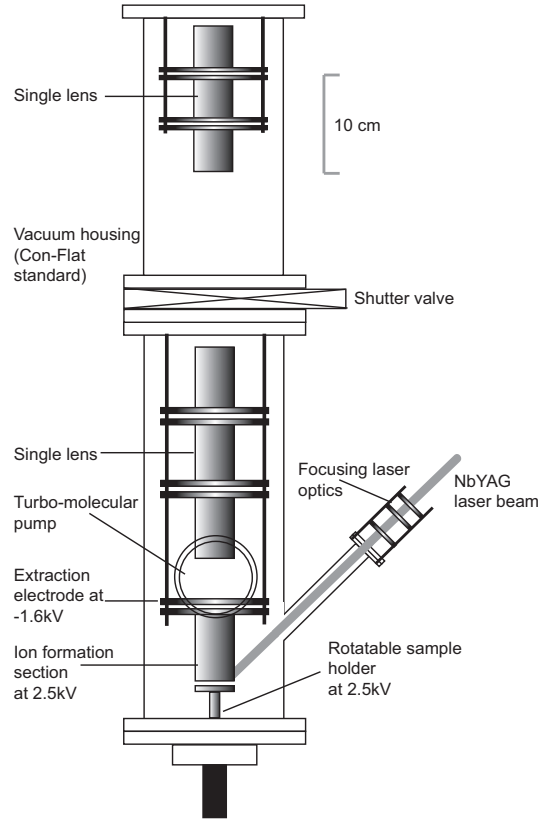

Fig. 16. Sketch of the laser ion source for producing ${ }^{12} \mathrm{C}_{n}^{+}$ clusters for mass references.

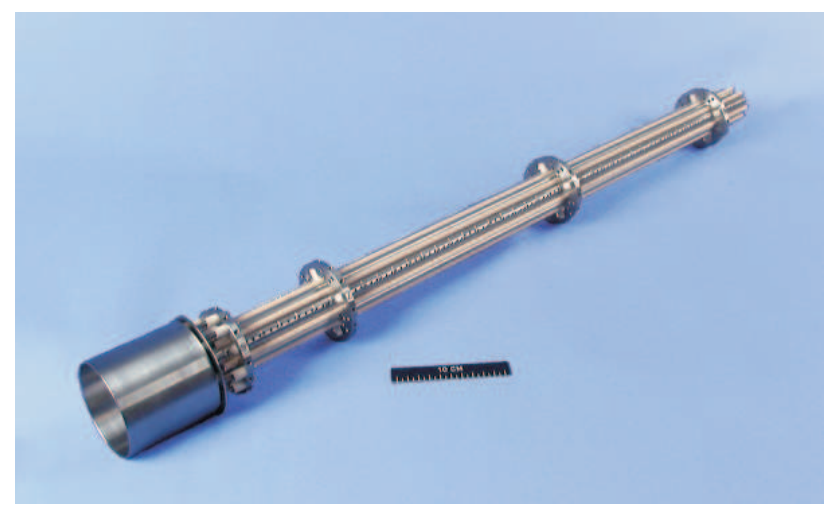

Fig. 17. Photograph of the radiofrequency quadrupole trap used as ion beam cooler and buncher at ISOLTRAP. The electrodes are made of stainless steel and the isolators of glass ceramic (Macor). The indicated scale is $10 \mathrm{~cm}$ long.

detail in $[27,41]$. From 1998 to 2000, i.e. before the realization and implementation of the present linear RFQ cooler and buncher, ISOLTRAP used a hyperboloid Paul trap to cool and bunch the ion beam from ISOLDE [67]. The main drawback of that trap was its low acceptance as compared to the large emittance of the ISOLDE beam. Therefore its capture efficiency was too low to be efficiently used. The present RFQ cooler and buncher along with its specially designed entrance electrode serve the purpose of better beam acceptance. Here, only the setup and its present performance will be presented.

The $60 \mathrm{keV}$ ISOLDE beam with transversal emittance of about $35 \pi \mathrm{mmmrad}$ [27] is first decelerated electrostatically by an eggcup-shaped electrode (see fig. 18) to achieve a theoretical capture efficiency of about $35 \%$ into 
Table 1. Typical physical dimensions and operational parameters of the RFQ cooler and buncher for ions in the mass regions $A \approx 39$ and $A \approx 133$ [27]. Electrode voltages marked with an asterisk $\left(^{*}\right)$ are given relative to $30 \mathrm{kV}$ or $60 \mathrm{kV}$, which corresponds to the potential of the ISOLDE ion source. The name of the electrodes are as shown in fig. 13 and fig. 18.

\begin{tabular}{|c|c|c|c|}
\hline Parameter & & Values & Dimensions / mm \\
\hline Helium pressure in RFQ $p_{\mathrm{He}}$ & & $\sim 10^{-3} \mathrm{mbar}$ & \\
\hline $\mathrm{RF}$ frequency $\nu_{\mathrm{RF}}=\omega_{\mathrm{RF}} / 2 \pi$ & & $990 \mathrm{kHz}$ & \\
\hline \multirow{2}{*}{$\mathrm{RF}$ amplitude $U_{\mathrm{RF}}$} & for $A \approx 39$ & $97 \mathrm{~V}$ & \\
\hline & for $A \approx 133$ & $135 \mathrm{~V}$ & \\
\hline \multirow{2}{*}{ Cooling time $T_{\text {cool }}$} & for $A \approx 39$ & $5 \mathrm{~ms}$ & \\
\hline & for $A \approx 133$ & $10 \mathrm{~ms}$ & \\
\hline Cage voltage: $U_{\mathrm{HV}}$ & & 30 or $60 \mathrm{kV}$ & \\
\hline Deceleration electrode* & & -1350 or $-3000 \mathrm{~V}$ & \\
\hline Focusing electrode* & inner diameter & -230 or $-180 \mathrm{~V}$ & 6 \\
\hline \multirow[t]{13}{*}{ Quadrupole rod segment* } & $\begin{array}{l}\text { diameter / total length } \\
\text { distance opposite electrodes }\end{array}$ & & $\begin{array}{c}18 / 881.5 \\
12\end{array}$ \\
\hline & no. 1 & $-60 \mathrm{~V}$ & 20.5 \\
\hline & no. 2 & $-40 \mathrm{~V}$ & 20.5 \\
\hline & no. 3 & $-25 \mathrm{~V}$ & 41.5 \\
\hline & no. 4 to no. 19 & -10 to $-14 \mathrm{~V}$ & 41.5 \\
\hline & no. 20 & -10 to $-14 \mathrm{~V}$ & 20.5 \\
\hline & no. 21 & -10 to $-14 \mathrm{~V}$ & 41.5 \\
\hline & no. 22 & -10 to $-14 \mathrm{~V}$ & 10 \\
\hline & no. 23 (accumulation) & $-16.5 \mathrm{~V}$ & 10 \\
\hline & no. 23 (ejection) & $+2 \mathrm{~V}$ & 10 \\
\hline & no. 24, no. 25 & $-17.8 \mathrm{~V}$ & 10 \\
\hline & no. 26 (accumulation) & $0 \mathrm{~V}$ & 20.5 \\
\hline & no. 26 (ejection) & $-70 \mathrm{~V}$ & 20.5 \\
\hline Plates of the extraction system* & inner diameter & $-240 \mathrm{~V}$ & 6 \\
\hline Einzel lens system* & & $-95 \mathrm{~V}$ & \\
\hline Pulsed cavity* & & $-2740 \mathrm{~V}$ & \\
\hline Accumulation time & & 0.001 to $1000 \mathrm{~ms}$ & \\
\hline Cooling time & & 2 to $20 \mathrm{~ms}$ & \\
\hline
\end{tabular}

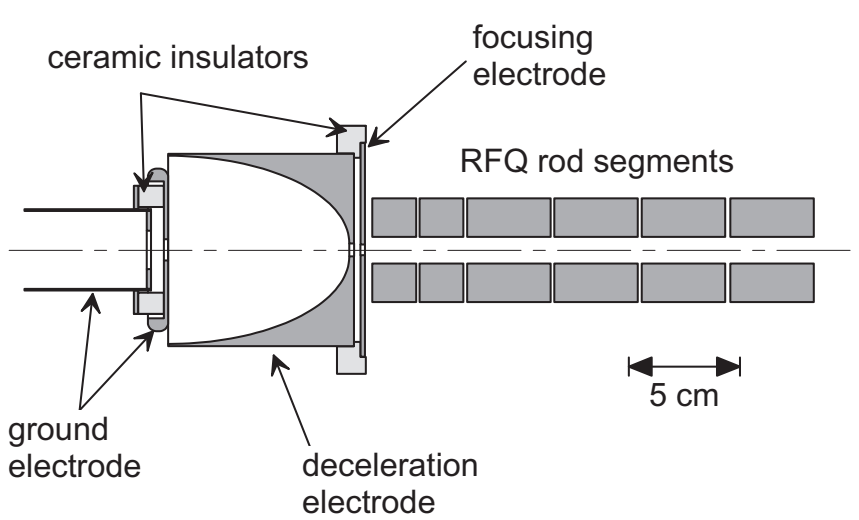

Fig. 18. The "eggcup"-shaped deceleration electrode to match the large emittance of the ISOLDE beam and the first rod segments of the radiofrequency quadrupole cooler and buncher $[27,68]$.

the RFQ structure situated in a high-voltage platform of $60 \mathrm{kV}$ [27]. The emittance of the ISOLDE beam is reduced by buffer gas cooling $\left(p_{\mathrm{He}} \approx 10^{-3}\right.$ mbar) inside the cooler and buncher. The voltages applied to the electrodes create a trapping potential as shown in fig. 2. The cooled

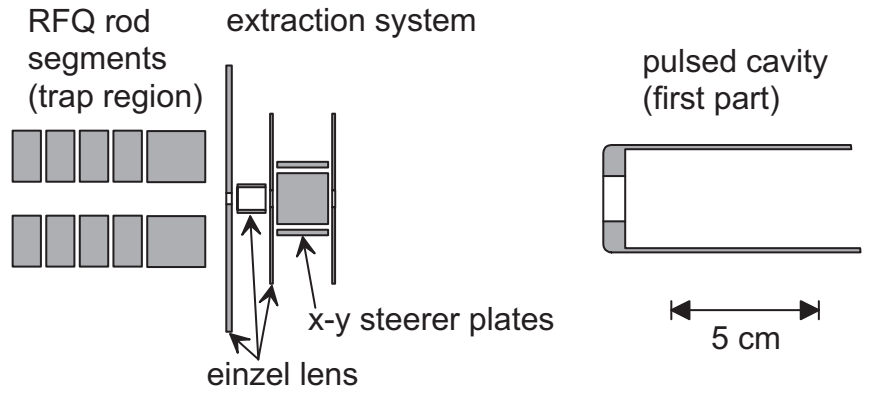

Fig. 19. A sketch of the extraction part of the RFQ cooler and buncher.

ion bunch is extracted after accumulation for a period of time between microseconds to several hundred milliseconds and a subsequent cooling time of $5-10 \mathrm{~ms}$ by switching the last electrodes. Beam optimization is possible via an einzel-lens system and a XY-steerer (see fig. 19). The cooled ion bunch is ejected from the $60 \mathrm{kV}$ RFQ with a temporal width of less than $1 \mu \mathrm{s}$. The physical dimensions and the normal operational parameters of the RFQ cooler and buncher are given in table 1. 
Table 2. Performance of the RFQ cooler and buncher of ISOLTRAP.

\begin{tabular}{ll}
\hline Parameters & Values \\
\hline Transversal emittance & $2 \pi \mathrm{mm}$ mrad at $60 \mathrm{keV}$ \\
Longitudinal emittance & $10 \mathrm{eV} \mu \mathrm{s}$ \\
Efficiency & $12-15 \%$ for Xe isotopes \\
\hline
\end{tabular}

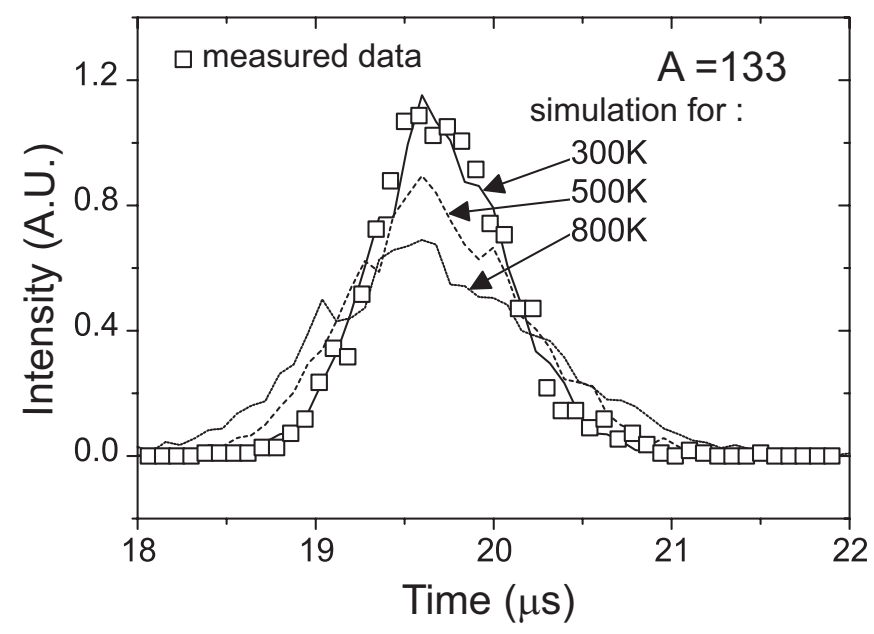

Fig. 20. Time-of-flight distribution of ${ }^{133} \mathrm{Cs}^{+}$ions ejected out of the RFQ cooler and buncher after an accumulation and cooling time of $20 \mathrm{~ms}$ in helium buffer gas at a pressure of $p_{\mathrm{He}} \approx 10^{-2}$ mbar at the trapping region. The ions are extracted at a kinetic energy of $30 \mathrm{keV}$. The solid lines show simulated values for the time-of-flight distribution under the same conditions but at different initial ambient temperatures.

The performance of the RFQ cooler and buncher is summarized in table 2 [27]. The cooling time mentioned above refers to the time needed to bring the ion cloud inside the RFQ to thermal equilibrium with the buffer gas at room temperature. The accumulation time can be varied depending on the half-life, the production rate, and the release of the ions from the ISOLDE target.

Figure 20 shows both the measured and the simulated time-of-flight distribution of ${ }^{133} \mathrm{Cs}^{+}$ions that are accumulated and cooled for $20 \mathrm{~ms}$ at a buffer gas pressure of $10^{-3}$ mbar in the RFQ. The measured data is qualitatively well described by an ion cloud at a temperature of $300 \mathrm{~K}$. The theoretical curves are normalized to same number of ions. Detailed description about the simulations can be found in ref. [27].

\subsection{Beam transport system}

The ion optical elements that transfer the ion bunches from the RFQ cooler and buncher to the preparation trap are shown schematically in fig. 13. In order to achieve a mass-independent transport only electrostatic ion optical elements are used. The cooled ion bunch is extracted by a set of electrodes, an einzel lens and a XY-steerer. The RFQ cooler and buncher including the entrance and the extraction electrodes are placed in a high-voltage cage at
$60 \mathrm{kV}$. The ion bunches extracted out of the buncher are guided to a drift tube with a length of $l=380 \mathrm{~mm}$ and put at a potential of $57.3 \mathrm{kV}$. While the ions fly through the tube its voltage is pulsed to the ground potential. The ions remain with a kinetic energy of $2.7 \mathrm{keV}$. The ion bunches are then guided by two einzel-lens systems (Lens2 and Lens3), three electric quadrupoles (QP60, QP1, QP2), and one XY-steerer (QS70) towards a $90^{\circ}$ deflector. The latter is composed of two electrostatic electrodes (split in two parts) for bending and one quadrupole (QP3), inserted between the two parts of the $90^{\circ}$ deflector, to be used in achromatic mode. After the bender the ISOLTRAP beam line continues in the vertical direction with two quadrupoles (QP4 and QP5), two XY-steerers, four lenses (Ionoptic A,B,C,D), one drift tube (Lower drift tube), and three retardation electrodes (Lower, Middle, and Upper retardation) to the lower endcap electrode of the preparation Penning trap (see fig. 13). This section contains another pulsed cavity which lowers the ion energy from $2.5 \mathrm{keV}$ to about $100 \mathrm{eV}$ for an efficient capture in the preparation Penning trap.

\subsection{Penning trap alignment}

Alignment of the magnetic field with respect to the trap axis is of major concern for efficient beam transportation. Here, we briefly describe the alignment procedure which is followed at ISOLTRAP. A tube inside the magnet bore is made of $316 \mathrm{LN}$ stainless steel with low susceptibility and no magnetic enclosures in order to keep high homogeneity of the magnetic field. This tube serves as an ion-optical bench and is thus precisely machined inside to hold the ion-optical elements. The elements are mounted on $1 \mathrm{~cm}$ thick discs made of Oxygen-Free HighConductivity (OFHC) copper, which themselves fit into the honed tube within $\pm 0.05 \mathrm{~mm}$ and hence assure good alignment of the optics. The tube itself is mounted in cardanic holders on both ends. In order to achieve precise and reproducible positioning inside the bore of the magnet, micrometer screws are used. The trap and all other ion optical elements were placed into the bore of the magnet only after an alignment of the vacuum tube with respect to the magnetic-field axis was performed. The tool for alignment consists of an electron gun, pin holes, and a number of diaphragms, all mounted on discs which fit with small tolerance to the vacuum tube. The electron gun, which is a current-heated filament with $0.2 \mathrm{~mm}$ pin holes on either side, was aligned with the tube axis. Two detector assemblies, each consisting of a $0.4 \mathrm{~mm}$ pinhole in the front and an electrically isolated plate on the back, were placed on the axis of the tube at a distance of about $25 \mathrm{~cm}$ from the electron gun. The whole assembly was inserted into the tube such that the electron gun sits in the center of the magnetic field. The electrons produced by heating the filament are guided by the magnetic field on either side. By careful positioning the tube with respect to the bore the electron beam passing through the pinholes of the detectors was maximized. 
After the vacuum tubes have been aligned to the magnetic field in both magnets, the two vacuum tubes must be aligned to the same optical axis. For this, the superconducting magnets must be shifted in $x y$-direction and tilted with respect to each other. To achieve this, the cryostats of the magnets are mounted on a special support structure which allows to individually move and tilt the magnets with respect to each other by the use of fine pitch threads. This alignment is performed and checked by using a theodolite.

\subsection{Beam monitors and detectors}

Single-ion detectors as well as devices for measurements of the ion current play an important role to monitor and optimize the whole beamline. In addition single-ion detectors are used finally to determine the ion's time of flight in order to perform the frequency measurements. To monitor the $60 \mathrm{keV}$ ISOLDE beam current and its profile, a Faraday cup detector and a scanner can be placed in front of the RFQ entrance. This ensures proper injection of the ISOLDE beam into the RFQ structure.

Another movable Faraday cup and an MCP detector (MCP1(h), see fig. 13) are installed on the ejection side of the RFQ. This allows one to optimize the ISOLDE beam transfer and RFQ injection by shooting through the RFQ structure. ISOLTRAP uses microchannel-plate (MCP) detectors to monitor the ion bunch transfer. Along the beam line there are seven movable MCP detectors (see fig. 1), four of them are shown in fig. 13. They are composed of two MCPs with either $50 \mathrm{~mm}$ (MCP5 vertical, which is not shown in fig. 13) or $25 \mathrm{~mm}$ active area mounted in a Chevron arrangement. No electron repelling grids are in use. The typical efficiency of such an MCP arrangement is about $30 \%$. The last MCP detector MCP5 is presently used as an alternative to a more efficient channeltron detector which is discussed below.

\subsection{The preparation Penning trap of ISOLTRAP}

The second trap of the ISOLTRAP setup is the preparation Penning trap. Since the last comprehensive review of the ISOLTRAP setup [14], the $0.7 \mathrm{~T}$ electromagnet was replaced by a superconducting magnet of $4.7 \mathrm{~T}$ (Oxford Instruments, System 200/130), which improved the performance of the cleaning procedure drastically [25]. The Penning trap itself was changed. It is a cylindrical Penning trap where the quadrupolar electric potential is improved by the use of correction electrodes (fig. 21). A nested potential well is formed by applying proper DC voltages to its 13 cylindrical segments [42]. The outer shape of this trap is optimized to capture ion bunches of several $10 \mathrm{eV}$ kinetic energy while the central part is carefully shaped to achieve a quadrupole potential over the largest possible volume. This and a large inner-trap radius of $20 \mathrm{~mm}$ allow for the acceptance of the incoming ion bunch and

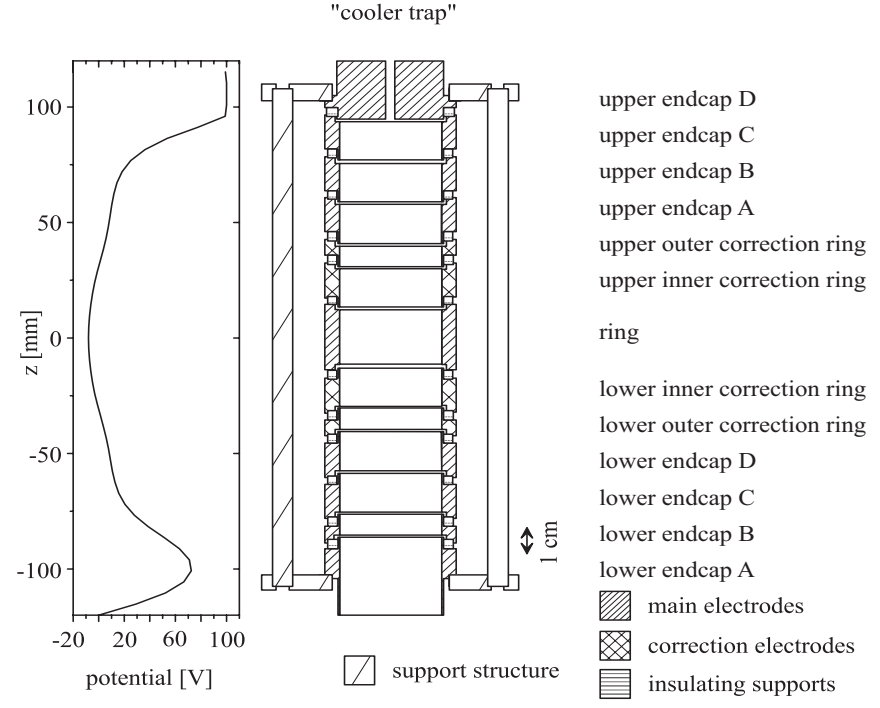

Fig. 21. Cut through of the cylindrical preparation Penning trap of ISOLTRAP including the shape of the trapping potential along the magnetic-field axis.

to cool and store more than $10^{3}$ ions at a time. The center electrode is eightfold segmented in order to apply a quadrupolar or even an octupolar RF field for excitation of the ion's radial motion. The mass-selective buffer gas cooling technique [25], as described in sect. 2.4.3, is applied here at a helium buffer gas pressure of $10^{-3}-10^{-4}$ mbar.

The ion beam cooling/selection is performed in three steps. The ions after being trapped lose their residual axial energy within tens of milliseconds by buffer gas collisions as explained in sect. 2.1 (axial cooling). A dipolar $\mathrm{RF}$ excitation at the magnetron frequency is applied for about $100 \mathrm{~ms}$ to drive the ion cloud to a magnetron radius larger than the diameter of the diaphragm $(3 \mathrm{~mm})$ at the exit of the trap (upper endcap D). To bring back the wanted ion species towards the trap center (sect. 2.4.3), a quadrupolar RF excitation at the true cyclotron frequency is applied before they are ejected out of the trap after a certain delay time. The RF excitation times vary according to the applied RF amplitude and the helium pressure as explained in sect. 2.4.2. Therefore, a procedure as described in refs. $[25,42]$ is adopted for optimizing the RF amplitudes, times, and the buffer gas pressure.

A mass-resolving power in the order of $10^{4}$ to $10^{5}$ [42] is routinely achieved within the new preparation Penning trap. This is sufficient to separate different isobars from a mixture (see fig. 22). However, note that the two resonance peaks at higher frequencies have larger width (more than $40 \mathrm{~Hz}$ ) compared to the resonance of the reference ion (about $30 \mathrm{~Hz}$ ), indicating some unresolved isobars.

The trap parameters are listed in table 3 . The alignment of the trap axis with respect to the magnetic-field axis is achieved by following the procedure as described in sect. 3.5. Two apertures of $3 \mathrm{~mm}$ diameter on the upper endcap D of the trap (fig. 21) serve for differential pumping. As discussed in sect. 2.6, space charge effects shift the cyclotron frequency due to Coulomb interaction between 
Table 3. The dimensions and the static and dynamic parameters for the preparation Penning trap under normal trapping and ejection conditions. The distance between neighboring trap electrodes is $1 \mathrm{~mm}$.

\begin{tabular}{|c|c|c|c|}
\hline Element & Length $(\mathrm{mm})$ & Radius (mm) & Voltage (V) for trapping/ejection \\
\hline Lower endcap A & 36.0 & 20.0 & $+88.5 /+10$ \\
\hline Lower endcap B & 9.0 & 20.0 & $+14 /+20$ \\
\hline Lower endcap C & 17.0 & 20.0 & $+10 /+20$ \\
\hline Lower endcap D & 17.0 & 20.0 & $+10 /+20$ \\
\hline Lower outer correction ring & 9.0 & 20.0 & $+3.5 /+5.2$ \\
\hline Lower inner correction ring & 16.6 & 20.0 & $-6.6 /+1.5$ \\
\hline Ring electrode & 23.8 & 20.0 & $-10.0 /-10.0$ \\
\hline Upper inner correction ring & 16.6 & 20.0 & $-6.6 /-19.6$ \\
\hline Upper outer correction ring & 9.0 & 20.0 & $+3.5 /-24.7$ \\
\hline Upper endcap A & 17.0 & 20.0 & $+10.0 /-30.0$ \\
\hline Upper endcap B & 17.0 & 20.0 & $+10.0 /-39.0$ \\
\hline Upper endcap C & 17.0 & 20.0 & $+10.5 /-45.0$ \\
\hline Upper endcap D & 25.0 & 1.5 & $+100.0 /-50.0$ \\
\hline Characteristic trap dimension $d$ & & & $31.7 \mathrm{~mm}$ \\
\hline \multicolumn{4}{|l|}{ Magnetic field } \\
\hline Field strength at the trap center & & & $4.7 \mathrm{~T}$ \\
\hline Relative homogeneity & & & $<10^{-6} / \mathrm{cm}^{3}$ \\
\hline Relative stability & & & $<10^{-8} / \mathrm{h}$ \\
\hline \multicolumn{4}{|l|}{ Vacuum } \\
\hline Without buffer gas & & & $5 \times 10^{-8} / \mathrm{mbar}$ \\
\hline With buffer gas (He) & & & $10^{-3}-10^{-4} / \mathrm{mbar}$ \\
\hline Typical eigenfrequencies for an ion with & $A=100$ & & \\
\hline$\nu_{+}$ & & & $728 \mathrm{kHz}$ \\
\hline$\nu_{-}$ & & & $0.3 \mathrm{kHz}$ \\
\hline$\nu_{z}$ & & & $21 \mathrm{kHz}$ \\
\hline
\end{tabular}

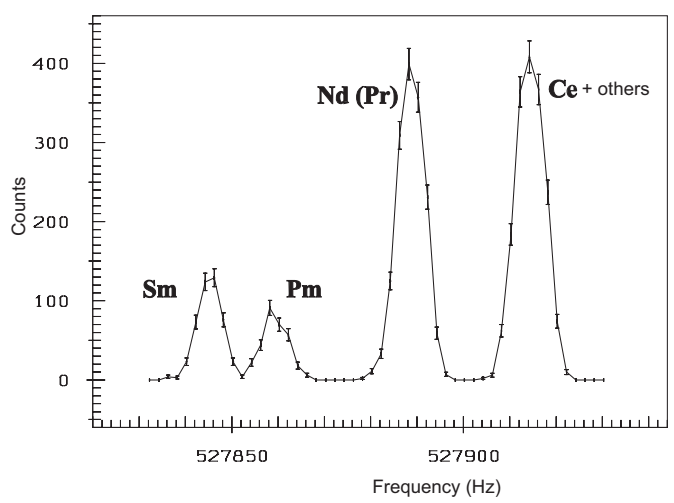

Fig. 22. Ion counts as a function of the quadrupolar excitation frequency. The graph shows resonances at the cyclotron frequencies for different isobars of mass number $A=138$ which are clearly resolved [69].

two different ion species trapped simultaneously in the trap. This shift increases with the number of stored ions. Such effects have been investigated in the preparation trap of ISOLTRAP for ${ }^{39} \mathrm{~K}$ and ${ }^{41} \mathrm{~K}$ ions [66] provided by the off-line alkali surface ion source. After isobaric cleaning in the preparation trap, which lasts between $30 \mathrm{~ms}$ and several $100 \mathrm{~ms}$ depending on the required resolving power and the half-life of the radionuclide under investigation, the purified ion bunch is transported to the precision Penning trap.

\subsection{The mass spectrometer section of ISOLTRAP}

The precision mass spectrometer of ISOLTRAP consists of three parts: an ion transport system from the preparation Penning trap to the precision Penning trap, the precision Penning trap itself, and an ion drift region from the trap to the detector located outside the magnetic field. Figure 23 gives an overview of the ion optics as well as the vacuum system of this section. Even though many advancements have been made in the measurement technique over the last decade, most of the hardware of this section remained the same as reported in [14]. The purpose of this section is to measure the cyclotron frequency of a trapped ion in the precision trap by the TOF ion cyclotron resonance method as described in sect. 2.5. 


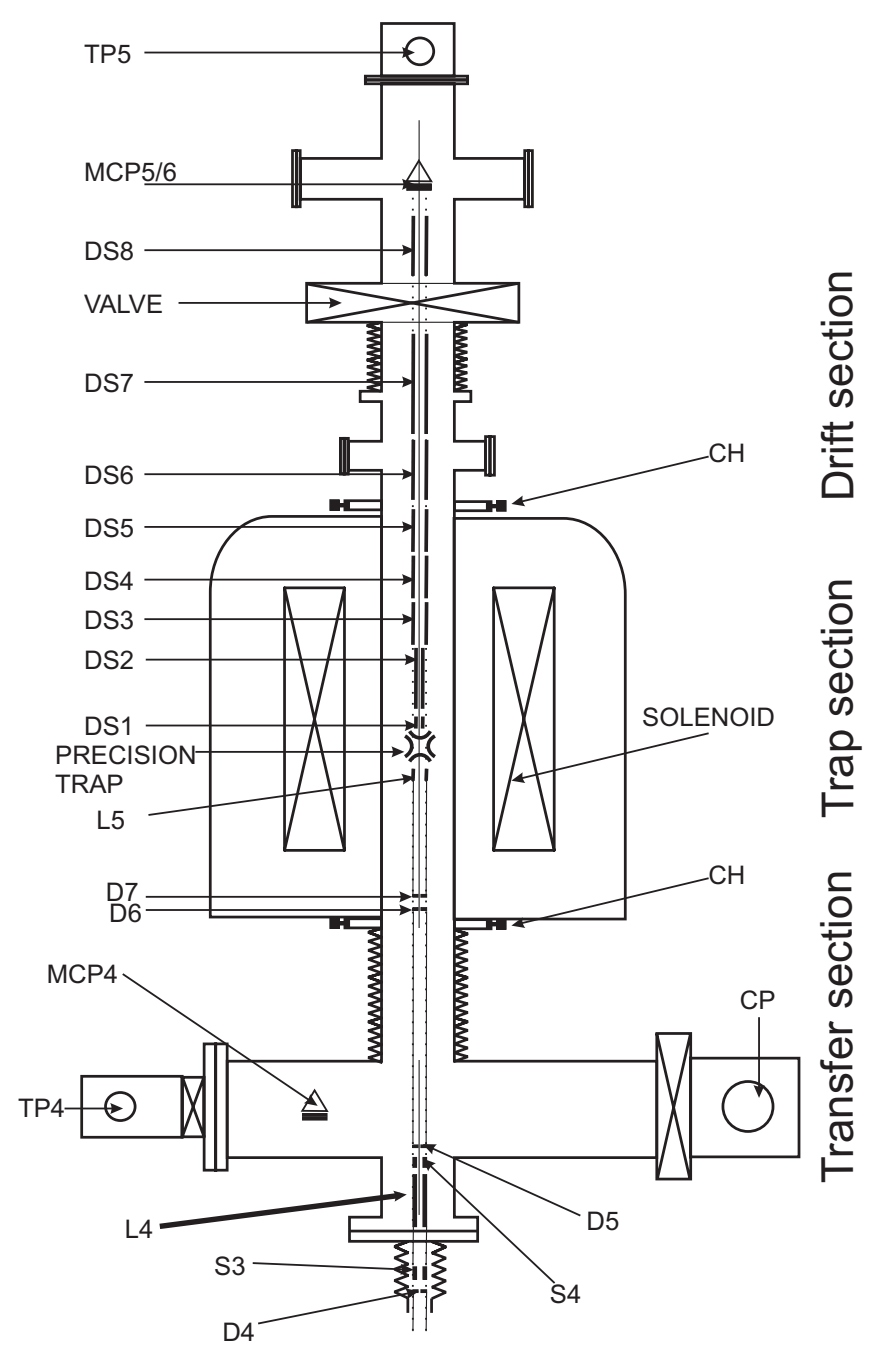

Fig. 23. Schematic layout of the spectrometer section of ISOLTRAP comprised of a transfer, a trap, and a time-of-flight drift section. $\mathrm{CH}$ denotes cardanic holder, CP cryopump, TP turbo pumps, D adjustable diaphragms, DS electrostatic elements, L lens, S steerers, and MCP micro-channel plate.

A warm-bore superconducting magnet (OXFORD $250 / 89$ ) with a bore diameter of $89 \mathrm{~mm}$ provides a magnetic field of $5.9 \mathrm{~T}$ for the precision trap [14]. The magnet is equipped with superconducting and room temperature shim coils in order to achieve a homogeneity of $\delta B / B<10^{-7}$ across a volume of $1 \mathrm{~cm}^{3}$. The temporal stability is $10^{-8} / \mathrm{h}$. The bore of the superconducting magnet housing the precision Penning trap has recently been equipped with a PID (Proportional Integration Differential) controlled temperature stabilization. This device provides a temperature stability of about $\pm 10 \mathrm{mK}$ per day and works on the principle of blowing in air at a wellcontrolled temperature. There is no cooling possible with the present system. In a similar way a pressure stabilization for the liquid-helium flow to the recovery line has been implemented with a stability of better than \pm 0.1 mbar per day [70]. This allows to minimize the magnetic-field fluctuations.
The spectrometer section consists of three vacuum chambers [14]: one below the magnet, a tube holding the precision trap inside the bore of the magnet, and the detector chamber placed above the magnet. These chambers are connected by bellows in order to ease the alignment. All vacuum parts are made of stainless steel. An ultra-high vacuum of $p<10^{-8}$ mbar is achieved with turbomolecular pumps at both, the lower (TP4) and the detector chamber (TP5), and a cryopump (CP) at the lower chamber. As mentioned in sect. 3.5, the cardanic holders (see fig. 23) with micrometer screws allows the alignment of the trap with respect to the magnetic-field axis.

From the geometry of the alignment setup it can be deduced that the tilting angle of the tube with respect to the magnetic-field axis is as small as $\theta$ (in degree) $\leq$ $1 \times 10^{-3}$ [14]. Considering the present ISOLTRAP precision Penning trap parameters this tilting angle can lead to a maximum frequency shift of $\Delta \nu^{\text {tilt }}=\Delta \omega^{\text {tilt }} /(2 \pi)<$ $2 \mathrm{mHz}$ [14]. This results in a maximum relative calibration error for ${ }^{133} \mathrm{Cs}$ of less than $2 \times 10^{-9}$.

\subsubsection{Ion transfer section}

The purpose of the ion transfer section [14] is to accept the ion bunch prepared and delivered by the preparation trap and to transport it to the precision trap. The ion optical system (see fig. 23) consists of four drift tubes (DS4-DS7), two sets of steerers $(\mathrm{S} 3, \mathrm{~S} 4)$ placed in the low magneticfield region, a set of retardation electrodes (L5) in front of the trap, and an einzel lens (L4). A segmented microchannel-plate detector (MCP4) in front of the precision trap allows one to monitor the ion transfer.

This transfer section is important in terms of proper and efficient injection into the high magnetic field. Improperly injected ions pick up high radial energies while passing through the magnetic-field gradient of the superconducting magnet. In extreme cases they are reflected by the high magnetic field (magnetic mirror effect). Since the cyclotron resonance detection scheme relies on the proper conversion from magnetron to cyclotron motion with the corresponding radial energy, it is essential to keep the initial radial energy as small as possible. Calculated ion trajectories for different injection conditions, i.e. different foci, show that the best injection focus lies $20 \mathrm{~cm}$ below the center of the magnetic field [14]. Under this condition practically no radial energy is picked up while the ions pass into the high magnetic-field region. For that purpose an einzel lens system and a set of steerers are used.

\subsubsection{The precision Penning trap of ISOLTRAP}

This trap has been designed to have minimum electric and magnetic-field imperfections over a large volume [14]. This is essential since the cyclotron resonance detection scheme needs to have the ions prepared at large radii in the order of $0.7-1 \mathrm{~mm}$. The choice was a highly compensated hyperboloid trap, which offers the best compromise between a perfect field and a desirable trap size. Figure 24 shows 


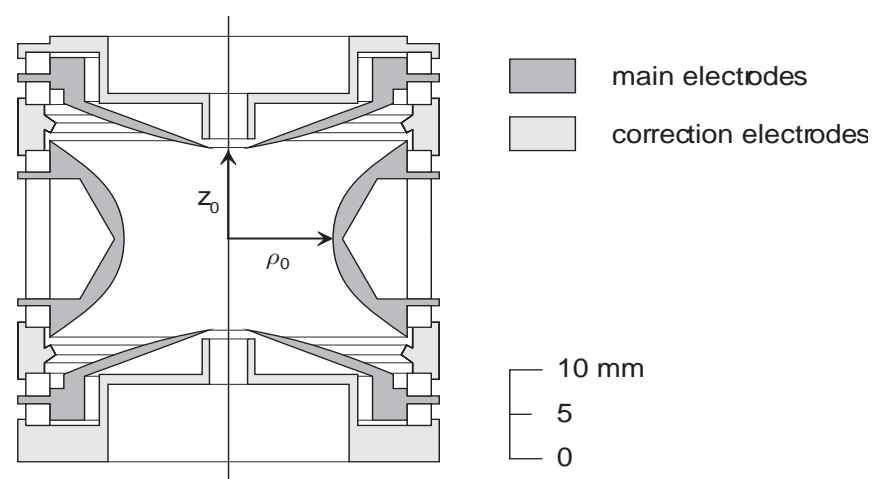

Fig. 24. Sketch of the ISOLTRAP precision Penning trap. Correction elements are in light grey, main electrodes in dark grey. Here $\rho_{0}$ and $z_{0}$ are the distance from the trap center to the ring electrode and the endcap electrodes, respectively.

Table 4. The dimensions, static and dynamic parameters for the precision Penning trap of ISOLTRAP under normal trapping/ejection conditions. The trapping voltage for this set of parameters is $U_{0}=8.4 \mathrm{~V}$.

\begin{tabular}{lrl}
\hline Element & $\begin{array}{c}\text { Trapping } \\
\text { voltage }(\mathrm{V})\end{array}$ & $\begin{array}{l}\text { Ejection } \\
\text { voltage }(\mathrm{V})\end{array}$ \\
\hline Lower correction tube & +1.1 & +1.2 \\
Lower endcap & -1.6 & -1.6 \\
Lower correction electrode & -6.6 & -6.6 \\
Ring electrode & -10.0 & -2.5 \\
Upper correction electrode & -6.6 & -6.6 \\
Upper endcap & -1.6 & -2.6 \\
Upper correction tube & +1.1 & -2.6 \\
\hline \hline
\end{tabular}

Characteristic trap dimension

$\rho_{0}$

$13.00 \mathrm{~mm}$

$z_{0}$

$11.18 \mathrm{~mm}$

$d$

$10.23 \mathrm{~mm}$

Magnetic field

Field strength at the trap center $5.9 \mathrm{~T}$

Relative homogeneity

Relative stability

$<10^{-7} / \mathrm{cm}^{3}$

Vacuum $<10^{-8} \mathrm{mbar}$

Typical eigenfrequencies for an ion with $A=100$

$\nu_{+}$

$910.0 \mathrm{kHz}$

$\nu_{-}$

$1.08 \mathrm{kHz}$

$\nu_{z}$

$44.0 \mathrm{kHz}$

the cross-sectional view of the trap, while its important parameters and dimensions are given in table 4 .

Deviations from the ideal quadrupole field are compensated by the correction electrodes installed between the endcap and the ring electrodes (for the finite size of the trap) and at the entrance and exit holes of the endcaps. To determine the required voltages for the correction electrodes, calculations were performed with the optimized re-

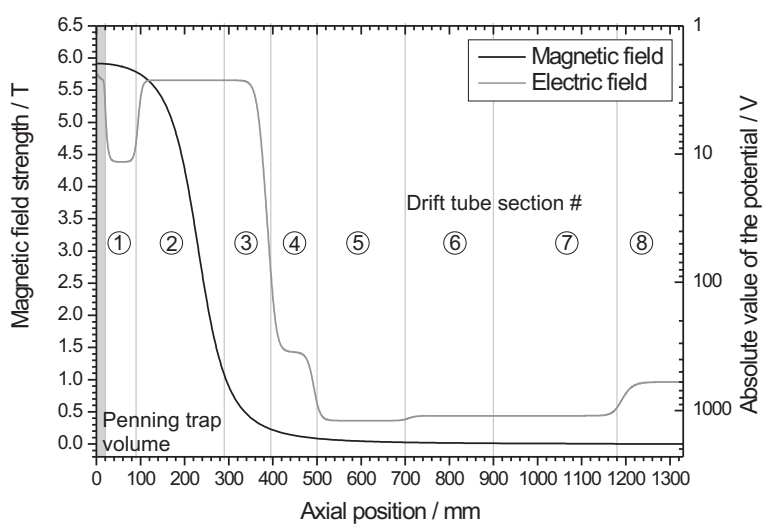

Fig. 25. The electric potential and the magnetic-field strength along the geometrical axis in the first part of the drift section of the mass spectrometer. Maximum retardation is applied in the region of the strongest magnetic-field gradient in order to achieve a large time-of-flight effect [71].

laxation method, which was found to give necessary precision, followed by a least-square fit to a pure quadrupole field, i.e., taking into account the sum of all higher multipoles. The octupole coefficient for the trap is found to be $\left|C_{4}\right| \approx 10^{-5}$ [14]. The calculations also give the best theoretical values for the voltages of the correction electrode. For a typical trapping voltage of $U_{0}=8.4 \mathrm{~V}$, the experimentally determined voltages are tabulated in table 4 . The values are in agreement with the calculated ones within the numerical accuracy of the calculation and the mechanical tolerance of the trap.

The ring electrode is split into four $90^{\circ}$ segments in order to apply the azimuthal quadrupolar RF field needed for the conversion of the ion motion. In order to preserve the good quality of the electric trapping field the slits are only $0.2 \mathrm{~mm}$ wide.

Magnetic-field inhomogeneities from the trap itself are kept small by the use of materials with low susceptibilities. The trap material is oxygen-free copper, which is first silver and then gold plated to prevent patch oxidation of the surface. Glass ceramics (MACOR $囚$ ) are used for insulation. In addition, all parts are machined as thin as possible in order to minimize magnetic-field distortions.

\subsubsection{The time-of-flight detection section}

The time-of-flight ion cyclotron resonance detection technique, as discussed in sect. 2.5, allows one to measure the difference in the ion's time of flight from the trap to the detector as a function of the excitation frequency. For this an appropriate drift section is designed, which enables to fully convert the initial radial energy of the ions to axial energy. In addition, it serves to efficiently transport the ions to the detector situated $1.2 \mathrm{~m}$ above the trap outside the strong magnetic field. Figure 25 shows the magnetic and electric potentials in this section. The voltages applied to the drift tubes are listed in table 5 . Ions ejected from the precision trap drift with a few eV kinetic energy in the homogeneous 
Table 5. The dimensions and applied voltages for the ion optics in the time-of-flight drift section. With the installation of the new detector system these values have changed slightly [71].

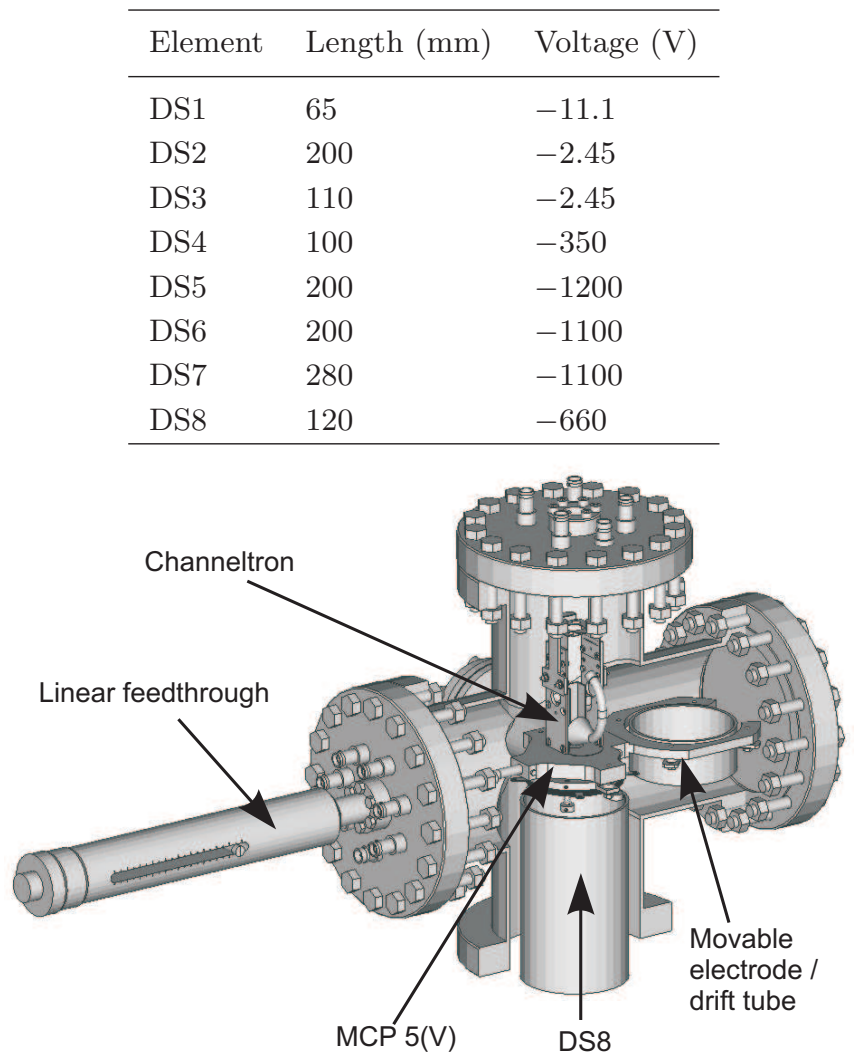

Fig. 26. Drawing of the new detector setup including a microchannel plate (MCP) and a channeltron detector. DS8 is the last electrostatic ion optical element before the detector, as shown in fig. 23.

part of the magnetic field at DS1 (see fig. 23). They are maximally retarded at the onset of the strongest gradient of the magnetic field and slowly drift through the highest field gradient (DS2,DS3) in order to convert $\approx 10 \mathrm{eV}$ radial energy fully into axial energy. In the second part of the TOF section the ions are accelerated (DS4,DS5,DS6) and focussed (DS7) to a micro-channel plate detector with $50 \mathrm{~mm}$ active area diameter.

In order to increase the detection efficiency and thus to minimize the required beamtime and to explore more exotic shorter-lived nuclides, the MCP detector for the time-of-flight ion cyclotron detection $(\mathrm{MCP} 5(\mathrm{v}))$ was recently replaced by an off-axis channeltron detector with conversion dynode. This detector provides close to $100 \%$ detection efficiency which will thereby increase the accessibility to even shorter-lived nuclei [71]. A drawing of the new detection setup is shown in fig. 26. It allows to use the highly efficient channeltron detector while keeping the previous $\mathrm{MCP} 5(\mathrm{~V})$ detector as spare.

\subsection{Control system}

At ISOLTRAP more than 150 different parameters need to be controlled. The basic requirement for the control sys-

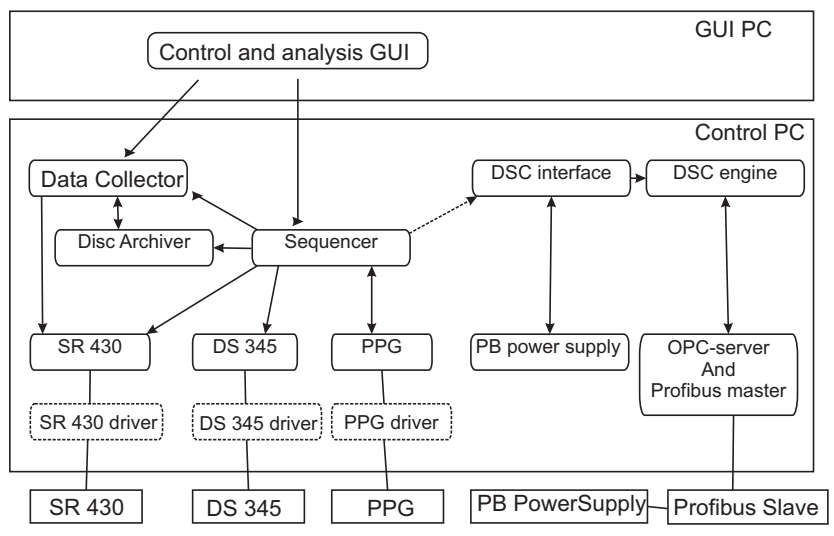

Fig. 27. Schematic layout of the ISOLTRAP control system. Rectangular boxes represent the hardware while roundcornered boxes represent the software. Active (inactive) software threads of execution have solid (dashed) boxes. Arrows indicate event-driven communication. The direction of the arrows do not indicate the direction of the data flow, but mark caller and callee. To simplify the figure, trending and alarming is indicated only for the Sequencer but not for the other active threads of execution on the Control PC (dashed arrow). Direct method calls and hardware connections are marked by lines. Uppercase and lowercase letters as well as numbers denote communication paths. For a detailed explanation see [72].

tem is to run the measurement cycle (see sect. 4.1) with a time precision well below $1 \mu \mathrm{s}$. More importantly the system has to be synchronized to follow the time sequences repeatedly and reliably over a week of beamtime. Since spring 2003, ISOLTRAP is using a new state-of-the-art control system based on the Control System (CS) framework [72], which has been developed by the Division for Information and Experimental Electronics at GSI. CS is an object-oriented, multi-threaded, event-driven framework with Supervisory Control and Data Acquisition (SCADA) functionality. It allows one to implement distributed control systems by adding experiment specific add-ons. A comprehensive documentation of the control system can be found in [72]. Here, only a brief description will be given.

Figure 27 shows a simplified layout of the ISOLTRAP control system. The main essence of this system is a hierarchy which gives flexibility for changes. Any change in the devices will not affect the control system as a whole and it is easy to incorporate new devices without requiring a thorough knowledge of the system. The hierarchy is composed of the hardware devices, a control PC, and a Graphical User Interface (GUI) PC. The main difference to the earlier control system lies within the control PC. In the past it was a VME bus with a Motorola E6 $\mathrm{CPU}$ and the control-system software developed in GNU $\mathrm{C}$ language. The new control system uses only one rackmounted PC. All devices can be accessed by GPIB (General Purpose Interface Bus) or via PROFIBUS (a popular fieldbus). The connection to the RFQ cooler and buncher at the high-voltage platform is provided by optical links. The control and on-line analysis GUIs are written in Bor- 


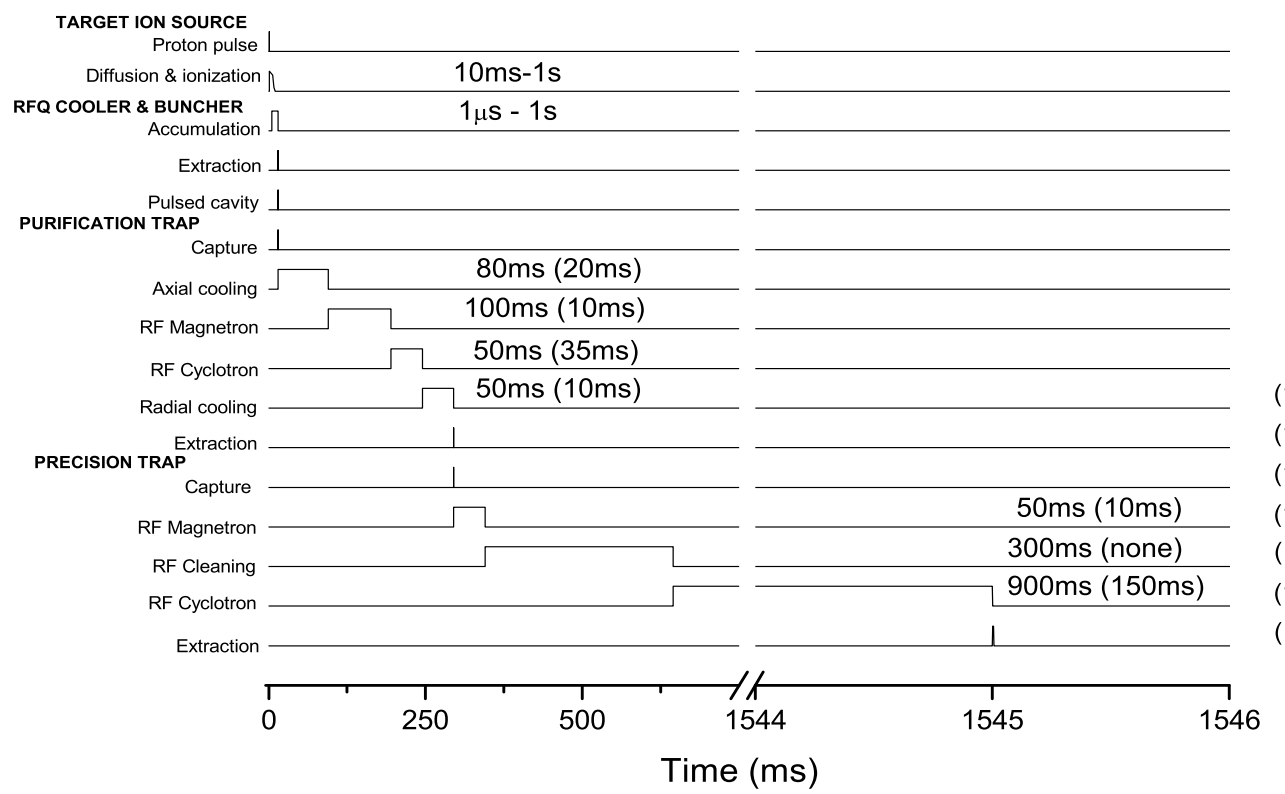

Fig. 28. Timing sequence of ISOLTRAP for mass
$100 \mathrm{~ms}$, values given in parenthesis) radionuclides.

land $\mathrm{C}++$ while the rest is based on the $C S$ framework, which itself is based on LabVIEW from National Instruments (NI). Typically 70 threads of execution are created for operating the experiment. For example, eight threads of execution are shown in fig. 27: $S R 430$ is a multi-channel scaler for data acquisition, DS345 is a function generator for the RF-excitation of the ion motion, $P P G$ is a pulse pattern generator to produce bit pattern with $100 \mathrm{~ns}$ precision, other devices like PB Power Supply, a power supply controlled by analog I/O of the profibus are described in [72]. The Sequencer is the heart of the control system maintaining the proper sequence of the experimental processes, the Data Collector serves to collect and buffer data from the acquisition devices, the Disc Archiver retrieves the buffered data from the Data Collector and writes it to a permanent storage device. The DSC Interface is the interface between all threads of execution and the $D S C$ Engine from NI. It serves for trending and alarming and is connected to the profibus master via OPC (Industrial standard for inter-process communication). Like the DSC Engine, the OPC server and the Profibus master are commercial products (Beckhoff).

\section{Experimental procedures}

Several different experimental mass measurement procedures and timing sequences are adopted, mainly depending on the nuclide of interest and the presence of impurities. In the following, the most commonly used procedure will be discussed. This sequence is carried out within $200 \mathrm{~ms}$ to $8 \mathrm{~s}$ depending on the half-life of the nuclide of interest. It includes accumulation, cooling, and bunching of the ISOLDE beam by the RFQ cooler and buncher, the purification and preparation of the ion ensemble in the preparation Penning trap, and finally the TOF ion cyclotron resonance measurement in the precision trap. This is one measurement cycle for a fixed frequency of the quadrupolar rf-excitation applied to the ions in the precision Penning trap. This cycle is repeated for different frequencies around the expected cyclotron frequency to obtain a TOF ion cyclotron resonance curve (see fig. 15(B)). The scan consisting of a certain number of frequency steps is repeated several times until the resonance is statistically convincing. This we call an ion cyclotron resonance (ICR) cycle. To perform a mass measurement, such an ICR cycle for the ions of interest is carried out in between two ICR cycles for the reference ion.

\subsection{Timing pattern of the experimental cycle}

Figure 28 shows the timing sequence for the cyclotron frequency determination of a radionuclide with half-life in the order of a few seconds, like ${ }^{22} \mathrm{Mg}\left(T_{1 / 2}=3.8 \mathrm{~s}\right)$ [73]. It also depicts within parenthesis the corresponding values for a mass measurement on a very short-lived radionuclide with a half-life of only about $100 \mathrm{~ms}$. The sequence is started by the proton impact (1) on the ISOLDE target. The diffusion and ionization of the radionuclides last between $10 \mathrm{~ms}$ and up to several seconds (2) depending on the release time of the radionuclide under investigation from the target. The processes in the RFQ buncher start with a certain delay (determined by the release time (2)), continues with the accumulation time of up to a few hundred ms followed by a short cooling time of 5-10 ms (3) and extraction (4). After a few $\mu$ s flight time, depending on the mass of the radionuclide, the pulsed cavities are switched down (5). The capture of the ion bunch in the preparation trap (6) is followed by an axial cooling by waiting for $80 \mathrm{~ms}(7)$, magnetron excitation for $100 \mathrm{~ms}(8)$, 
cyclotron excitation of $50 \mathrm{~ms}$ (9), radial cooling by waiting for $50 \mathrm{~ms}$ (10) before they are ejected out of the trap (11). A similar time pattern holds for the precision trap where capturing (12) triggers the dipolar magnetron excitation for $10 \mathrm{~ms}$ (13), followed by dipolar cleaning excitation (if needed) for about $300 \mathrm{~ms}$ (14) and finally the RF cyclotron excitation for $900 \mathrm{~ms}$ (15) before being ejected for the TOF measurement (16).

There are three parameters that determine the total duration of a measurement cycle: first, the required resolving power in the preparation trap; second, the envisaged resolving power and accuracy in the precision trap; and third, the half-life of the ions of interest. A longer buffer gas cooling in the preparation trap results in a higher resolving power for the mass-selective cooling process. Similarly, the narrower the line width of the TOF-ICR resonance in the precision trap, the more precise the center frequency can be determined. Of course the half-life gives a natural limit. ISOLTRAP uses short cycles for very shortlived radionuclides, e.g. for ${ }^{32} \operatorname{Ar}\left(T_{1 / 2}=98 \mathrm{~ms}\right)$ a total measurement cycle time of about $200-300 \mathrm{~ms}$ was used in order to minimize decay losses [74]. The exotic nuclide ${ }^{74} \mathrm{Rb}\left(T_{1 / 2}=65 \mathrm{~ms}\right)$ is the to date shortest-lived nuclide on which a high-precision mass measurement in a Penning trap has been performed [75]. In this case the cycle time was reduced to only $150 \mathrm{~ms}$.

\subsection{Ion selection and preparation}

The highest possible mass-resolving power of ISOLDE's high-resolution separator is at present about $m / \Delta m_{\text {FWHM }}=5000$. This can only be achieved by the use of slits after the separator magnet which also causes losses in the beam intensity [28]. In many cases this resolving power is not sufficient to obtain a pure mono-isobaric beam. Isobaric contaminants are the main impurities that need to be separated by the mass-selective buffer gas cooling in the preparation Penning trap. Here, the resolving power is limited to $10^{5}$ for masses around $A=100$ and for a cooling time of several hundred milliseconds. As long as the impurity-to-ion ratio is less than one, it is sufficient to remove all impurities. In cases where the ratio is much higher for a very specific isobaric impurity, a dipolar cleaning becomes essential after the mass-selective buffer gas cooling has been applied. Even this technique is only applicable for the ratios up to the order of $300: 1$ [66].

The isobarically purified ions are transferred to the precision Penning trap where they are captured in flight [23]. The delay between the ejection of the ion bunch from the preparation trap and its capture in the precision trap is very critical, since it may increase the ions energy if not properly selected (see below). The temporal spread of the ion bunch from the preparation trap to the measurement trap is about $1 \mu \mathrm{s}$ while the typical magnetron frequency is around one $\mathrm{kHz}$. So the ion bunch can be considered as having the same magnetron phase. As has been discussed in sect. 2.4.1, the delay between the ejection from the preparation trap and the capture in the precision trap is locked with respect to the phase of the applied magnetron RF excitation. This is known as the magnetron phase locking [26]. For a phase difference of $\pi / 2$ as was pointed out in sect. 2.4.1, the increase in the magnetron radii is maximum in minimal excitation time. Therefore, the magnetron phase locking mechanism is an efficient way to reduce the total cycle time and to pronounce the minimum in the time-of-flight spectrum. In the precision Penning trap an additional mass-selective cleaning using $\nu_{+}$excitation can be applied. In this way, the amount of contaminant ions can be decreased by at least an additional factor of 10 to 100 .

\subsection{Tuning of the trap parameters}

Section 2.6 summarized the importance to minimize the magnetic- and electric-field imperfections of the trap with the goal to achieve highest accuracy in direct mass determination. ISOLTRAP developed some optimization procedures to tune its precision trap and to determine the limit of the systematic uncertainties that are generated by the residual imperfections. More details on these issues may be found in $[76,77]$.

Once a proper transfer time is chosen, it is essential to minimize the magnetic-field inhomogeneities, as discussed in sect. 2.6. The transfer time is the tool to probe the extent of the homogeneous trap volume while the shift in the cyclotron frequency is the observable to indicate the magnetic-field homogeneity. The superconducting magnet of the precision trap has a set of 10 superconduction shim coils and one room temperature coil for shimming the $z^{2}$ component. First, the superconducting shim coils are shimmed using an NMR probe. This must be done before inserting the vacuum tube and the trap. Second, the $z^{2}$ shim component is shimmed by probing the magneticfield homogeneity with the ion's frequency shift. Figure 29 shows the cyclotron frequency $\nu_{c}$ as a function of the transfer time for different current values of the room temperature correction shim coils of the superconducting magnet. A current of $270 \mathrm{~mA}$ turns out to be the best value for which the cyclotron frequency varies only within the statistical limit with the transfer time. These measurements were performed with ${ }^{85} \mathrm{Rb}^{+}$ions from the reference surface ion source. However, the optimized value for the shim coil current is mass independent and thus can be kept for experiments with different masses.

In a very similar way as the magnetic-field optimization, the electric-field imperfections can be minimized by adjusting the voltages applied to the correction electrodes (correction rings as well as the correction tubes, see fig. 24) of the precision trap. Figure 30 shows a plot of the $\nu_{+}$ frequency, which is sensitive to the electric-field imperfections, for ${ }^{85} \mathrm{Rb}^{+}$as a function of the transfer time for different correction electrode voltages. The correction tube voltage of $1.120 \mathrm{~V}$ was found to be optimal since the reduced cyclotron frequency stays almost constant over a large variation of the transfer time, hence over a large trap volume. This optimization is performed after the magnetic-field optimization as this ensures the effect 


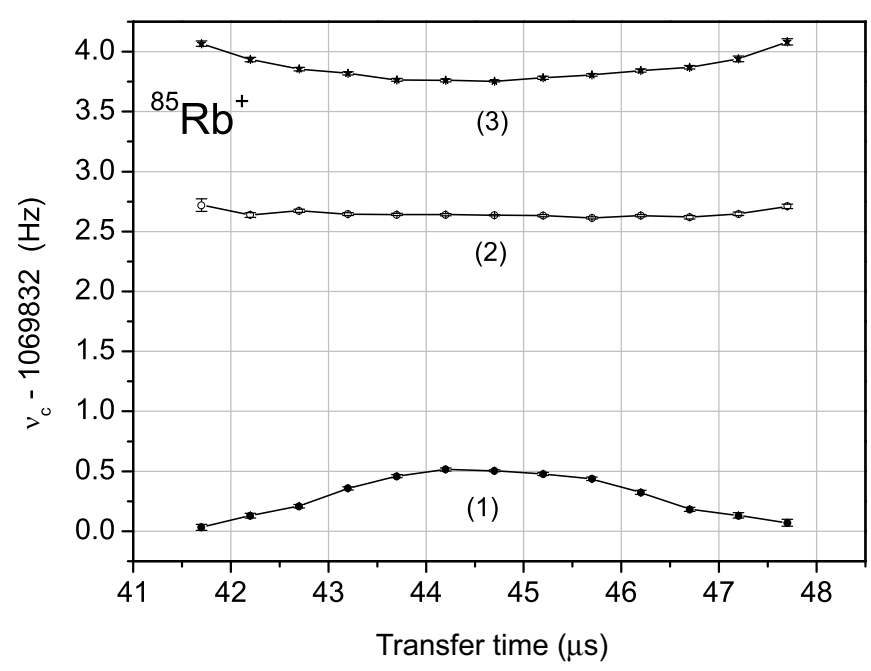

Fig. 29. Cyclotron frequency $\nu_{c}$ as a function of the transfer time in the precision trap for different values of the shim coil current [76]: (1) $100 \mathrm{~mA}$, (2) $270 \mathrm{~mA}$, and (3) $350 \mathrm{~mA}$. The optimal value was found to be $270 \mathrm{~mA}$.

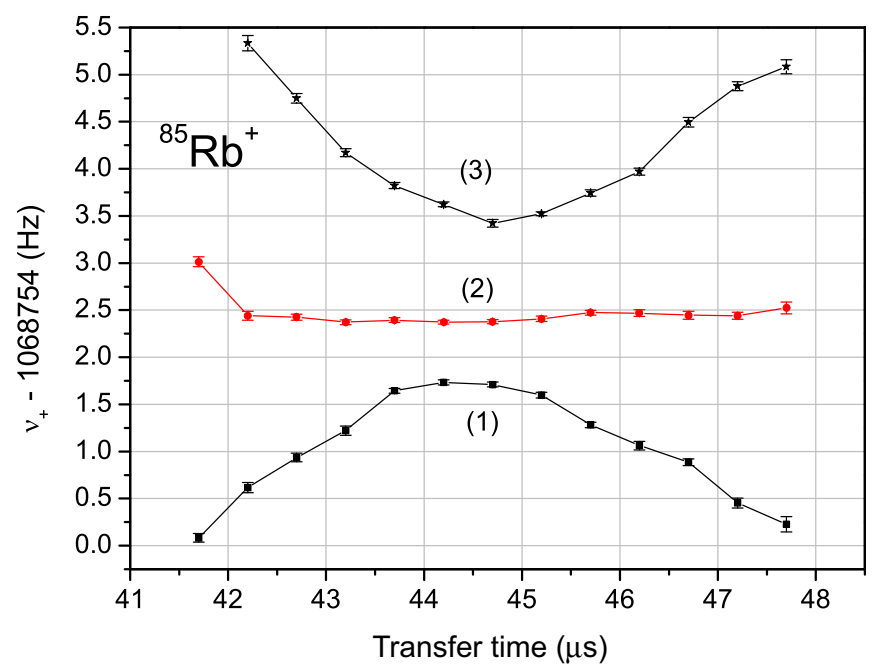

Fig. 30. Reduced cyclotron frequency $\nu_{+}$as a function of the transfer time in the precision trap for different values of the correction tube voltage [76]: (1) $1.250 \mathrm{~V}$, (2) $1.120 \mathrm{~V}$, and (3) $0.950 \mathrm{~V}$. The optimal value was found to be $1.120 \mathrm{~V}$.

of the magnetic-field inhomogeneity on the reduced cyclotron frequency to be negligible. More details on these optimization procedures can be found in $[76,77]$.

\section{Data analysis}

At ISOLTRAP we used carbon cluster ions from a laser desorption source to perform cross-reference mass measurements over a wide mass range from $A=72$ to $A=240$ [59]. A cross-reference mass measurement is a measurement which is carried out using carbon clusters both as the reference ion and as the ion of interest. In such a case, the value of the ratio of the cyclotron frequencies $\nu_{c, \text { ref }} / \nu_{c}$ is known with an uncertainty better than

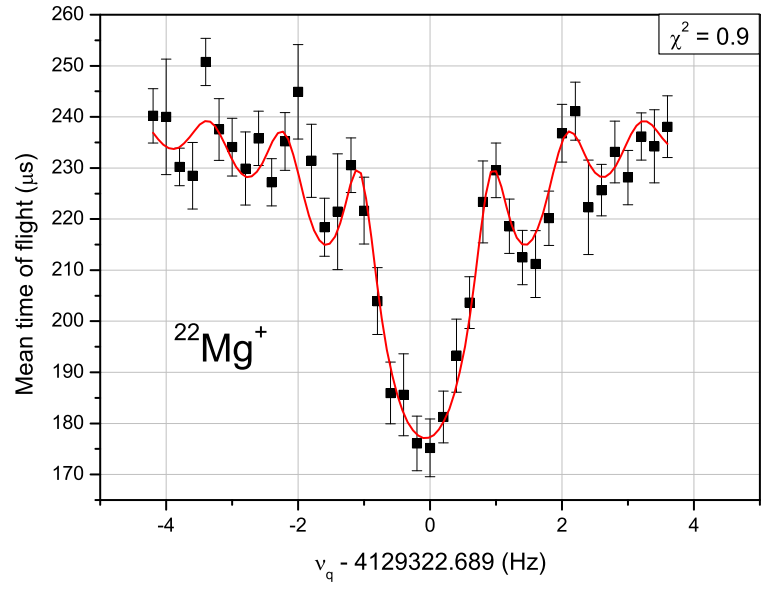

Fig. 31. The mean time of flight as a function of the quadrupolar rf-excitation frequency applied to the ring electrode segments of the precision trap. The resonance was obtained within 40 min during which about 350 ions in total were collected. The solid line is a least-square fit of the theoretically expected line shape [45] to the data points.

$10^{-10}$. These investigations thus helped in understanding the sources and to determine the extent of systematic uncertainties in measurements of unknown masses. Over a period of three months more than 350 cyclotron frequency resonances have been recorded. The evaluation of these data yielded the determination of the mass dependent systematic uncertainty, the steady decrease of the magnetic-field strength, the uncertainty due to magneticfield fluctuations, and the residual systematic uncertainty. A detailed description of the performed measurements can be found in $[46,59]$. Here, only the final results will be presented.

\subsection{Determination of cyclotron frequency ratios}

The typical approach adopted for ISOLTRAP data analysis will be briefly discussed here, with the mass measurement of ${ }^{22} \mathrm{Mg}$ taken as an example [78]. A number of well-known reference masses were used to calibrate the magnetic field. For simplification, the present discussion will be limited to ${ }^{39} \mathrm{~K}^{+}$as the reference ion. $\mathrm{A}^{22} \mathrm{Mg}^{+}$ TOF cyclotron resonance measurement recorded between two ${ }^{39} \mathrm{~K}^{+}$cyclotron resonance measurements completes one mass measurement cycle. Figure 31 shows an example of the mean TOF as a function of the quadrupolar rfexcitation frequency in the precision trap for ${ }^{22} \mathrm{Mg}^{+}$. The solid line is a least-square fit of the theoretically expected line shape [45] to the data points with $\chi^{2} \approx 1$. This fit determines the central frequency of the TOF resonance with its statistical uncertainty.

An analysis of the number of ions detected in each TOF measurement ( $z$-class analysis) [59] is used to take care of an eventual cyclotron frequency shift due to the presence of contaminant ions stored simultaneously in the precision trap. The data set is divided into subsets (classes) according to the average number of ions observed 


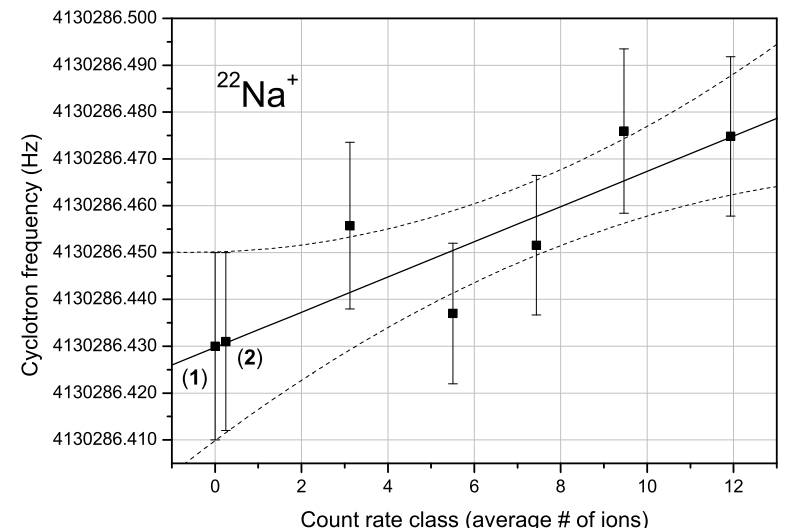

Fig. 32. Cyclotron frequency $\nu_{c}$ for ${ }^{22} \mathrm{Na}^{+}$as a function of the average number of detected ions ( $z$-class analysis, see text). The straight line is a linear least-squares fit to the data points. (1) and (2) are the extrapolated values for 0 and 0.25 average number of detected ions obtained from the fit. The dashed lines indicate the $1 \sigma$ confidence band of the fit.

by the TOF detector after ejection from the precision trap and TOF determination per measurement cycle. This number is proportional to the number of simultaneously stored ions in the trap. In the following, we discuss only the MCP detector with a detection efficiency of about $25 \%$. The cyclotron resonance frequencies determined for each class are plotted as a function of the average number of ions detected by the MCP detector. This is shown in fig. 32. Here, the radioactive nuclide ${ }^{22} \mathrm{Na}^{+}$is taken as an example because the count rate for ${ }^{22} \mathrm{Mg}^{+}$was below three per measurement cycle and hence a $z$-class analysis is not meaningful. A linear least-squares fit is used to extrapolate the frequency towards a single stored ion or no ions in the trap. A single stored ion corresponds to 0.25 average number of detected ions. In some cases, like that of ${ }^{22} \mathrm{Mg}$, the number of detected ions is less than three per ejected ion pulse from the precision trap. This number is too low to observe any frequency shift due to contaminations within the statistical uncertainties.

The ${ }^{22} \mathrm{Mg}^{39} \mathrm{~K}$ mass measurement cycle was repeated six times with about 350 ions for each ${ }^{22} \mathrm{Mg}^{+}$TOF resonance. Due to the low ${ }^{22} \mathrm{Mg}$ yield, which was further reduced by using the slits of the high-resolution mass separator (HRS) of ISOLDE, such measurement cycles took about $90 \mathrm{~min}$. To obtain the frequency ratio $r$, the two cyclotron frequencies of the reference ion $\left({ }^{39} \mathrm{~K}^{+}\right)$are then interpolated linearly to the time of the actual measurement of ${ }^{22} \mathrm{Mg}^{+}$[59]. The relative standard uncertainty due to the magnetic-field drift, calculated for the time interval between two reference measurements, is then added quadratically to the relative standard uncertainty in the cyclotron frequency determination for the reference mass.

The weighted mean $\bar{r}$ of the measured ratios of the cyclotron frequencies

$$
\bar{r}=\frac{\sum_{i} \frac{r^{i}}{u_{c}^{2}\left(r^{i}\right)}}{\sum_{i} \frac{1}{u_{c}^{2}\left(r^{i}\right)}}
$$

and its uncertainty

$$
\frac{u(\bar{r})}{\bar{r}}=\frac{1}{\bar{r} \sqrt{\sum_{i} \frac{1}{u_{c}^{2}\left(r^{i}\right)}}}
$$

are calculated, where $u_{c}\left(r^{i}\right)$ denotes the uncertainty of the $i$-th determination of the cyclotron frequency ratio after taking into account the uncertainty due to the magneticfield drift. For the measurements of ${ }^{22} \mathrm{Mg}$ with ${ }^{39} \mathrm{~K}$ as reference, $\bar{r}=0.5646108615$ and $\frac{u(\bar{r})}{\bar{r}}=1.3 \times 10^{-8}$ are obtained. This ratio is then corrected for the mass-dependent systematic error $u_{m}(r) / r$.

\subsection{Mass and time-dependent systematic errors}

From the measurement of the cyclotron frequency ratio between the ion of interest $(\nu)$ and the reference ion $\left(\nu_{\text {ref }}\right)$, the mass $m$ of the ion of interest can be derived using

$$
m=\frac{\nu_{\mathrm{ref}}}{\nu}\left(m_{\mathrm{ref}}-m_{e}\right)+m_{e},
$$

where $m_{\text {ref }}$ is a well-known reference mass and $m_{e}$ is the electron mass. Since ISOLTRAP measures the mass of singly charged ions, one has to correct for the missing electron mass while the binding energy of the electron is negligible. The final ISOLTRAP results are given as frequency ratios so that at any time the mass can be derived using the available best value of the reference mass. The uncertainties associated with the frequency ratio determination finally propagates to the mass uncertainties. As discussed in sect. 2.6, the known systematic uncertainties that are associated with ISOLTRAP are either massdependent or dependent on the temporal fluctuation of the magnetic-field amplitude. The mass-dependent error can be estimated from cross-reference mass measurements over a wide mass range.

The relative change of the magnetic-field magnitude in the precision trap can be determined by measuring the shift of the cyclotron frequency of a known mass over a long time period. The changes in the magnetic field are dependent on ambient conditions, like temperature, pressure, stray fields around the experiment, and presence of ferromagnetic materials near the magnet. Thus, it is difficult to keep it under full control. However, minimizing the time interval of the cyclotron frequency measurements between the reference ion and the ion of interest can reduce this systematic uncertainties significantly.

The variation of the relative deviation of measured cyclotron frequency ratios from that of the true value $e(r) / r$ as a function of the mass difference $m-m_{\text {ref }}$ was measured with different carbon cluster sizes [59]. Assuming linearity as a first-order effect, a mass-dependent systematic uncertainty,

$$
\frac{u_{m}(r)}{r}=-1.6(4) \cdot 10^{-10} / u \cdot\left(m-m_{\mathrm{ref}}\right),
$$

was obtained. Similarly, from the short-term magneticfield drift the time-dependent systematic uncertainty was 
determined to be

$$
\frac{u_{B}\left(\nu_{\mathrm{ref}}\right)}{\nu_{\mathrm{ref}}}=6.35(45) \cdot 10^{-11} / \mathrm{min} \cdot \Delta T \text {. }
$$

These sets of measurements were taken over a long time period but then divided into intervals of $15 \mathrm{~min}$, which is roughly the duration of ISOLTRAP's reference measurements. Incorporating both of these systematic uncertainties into the cross-reference carbon cluster measurements, the residual systematic uncertainty of ISOLTRAP is derived to be [59]

$$
\frac{u_{\mathrm{res}}(r)}{r}=8 \cdot 10^{-9}
$$

This uncertainty also represents the present limit of accuracy for cyclotron frequency ratio measurements with the ISOLTRAP Penning trap mass spectrometer because all other uncertainties, being statistical in nature, can be reduced by an increased number of repeated measurements. However, by measuring a local network of masses and using a least-squares analysis of all measured relations, the uncertainty limit can be smaller than the one given in eq. (33).

The difference in the reference mass and the mass of interest is $17 \mathrm{u}$ for the present example of ${ }^{22} \mathrm{Mg}$ mass measurement with respect to ${ }^{39} \mathrm{~K}$. This corrected value is then the final frequency ratio and is expressed as

$$
\bar{r}_{\mathrm{corr}}=\bar{r}\left[1+\frac{u_{m}(\bar{r})}{r} \cdot \Delta m\right] .
$$

Using eq. (31), we obtain $\bar{r}_{\text {corr }}=0.5646108630$. The final relative uncertainty of the frequency ratio is calculated by quadratically adding the above mentioned relative massdependent systematic uncertainty $u_{m}(\bar{r}) / \bar{r}$ and the relative residual systematic uncertainty $u_{\text {res }}(\bar{r}) / \bar{r}$ to the statistical uncertainty,

$$
\frac{u_{c}(\bar{r})}{\bar{r}}=\sqrt{\left[\frac{u(\bar{r})}{\bar{r}}\right]^{2}+\left[\frac{u_{m}(\bar{r})}{\bar{r}}\right]^{2}+\left[\frac{u_{\mathrm{res}}(\bar{r})}{\bar{r}}\right]^{2}},
$$

which turns out to be $1.3 \cdot 10^{-8}$. The mass of ${ }^{22} \mathrm{Mg}$ is then derived from the best known value of the mass of ${ }^{39} \mathrm{~K}$ [1], using eq. (30). Here, the obtained mass value is $m\left({ }^{22} \mathrm{Mg}\right)=21.99957090(30) \mathrm{u}$. More detailed information on these measurements and analysis can be found in [73].

\section{Performance of the ISOLTRAP mass spectrometer}

During the last 12 years, the performance of the Penning trap mass spectrometer ISOLTRAP with respect to efficiency, accuracy, accessible half-life, and resolving power has been considerably improved. These breakthroughs in mass spectrometry of radionuclides are briefly described in the following.

\subsection{Absolute mass measurements}

Carbon clusters provide ideal mass references for absolute mass measurements: They eliminate by definition the uncertainty of the mass of the reference ion. The molecular binding energies of the carbon clusters, being of the order of $5 \mathrm{eV}$, can be neglected at the present level of ISOLTRAP's precision. To perform absolute mass measurements at ISOLTRAP, a carbon cluster reference ion source has been developed and implemented (see sect. 3.2.2).

\subsection{Efficiency}

The total efficiency $\epsilon_{\text {tot }}$ of the ISOLTRAP apparatus can be expressed as

$$
\epsilon_{\mathrm{tot}}=\epsilon_{\mathrm{cap}} \cdot \epsilon_{\mathrm{bun}} \cdot \epsilon_{\mathrm{trans}} \cdot \epsilon_{\mathrm{det}}
$$

where $\epsilon_{\text {cap }}, \epsilon_{\text {bun }}, \epsilon_{\text {trans }}$, and $\epsilon_{\text {det }}$ are the capturing, bunching, transfer, and detection efficiencies at different sections of the apparatus. Since for the two Penning traps the capturing and trapping efficiencies are close to unity and the transfer efficiency is close to $90 \%, \epsilon_{\text {tot }}$ is mainly determined by capturing and bunching efficiency of the RFQ cooler and buncher as well as the detection efficiency. The installation of the RFQ cooler and buncher as well as a realignment of the setup resulted in an increase of the overall efficiency of the ISOLTRAP spectrometer to about 1-5\% for stable or long-lived nuclides, which is about two orders of magnitude higher than the performance in 1996 [14]. This efficiency as determined in November 2001 from the ratio of the number of ${ }^{36} \mathrm{Ar}$ ions observed by the MCP5 detector to the number of ions measured at the focal plane of the ISOLDE separator, includes the ISOLDE beamline transport efficiency which is about $80 \%$. For very shortlived nuclides such as ${ }^{32} \mathrm{Ar}$ with $T_{1 / 2}=98 \mathrm{~ms}$, an overall efficiency of about $0.1 \%$ was achieved, mainly due to additional decay losses [74]. The overall efficiency has recently been further improved to about $10 \%$ by careful tuning of the setup and the installation of a new highly efficient detector system, as mentioned in sect. 3.8.3.

\subsection{Accessible half-life}

As discussed in sect. 4.1, the total time needed for a measurement cycle is mainly given by the duration of the rfexcitations needed for cleaning and centering the ions in the preparation trap and for radiofrequency excitation in the precision trap. The reduction of the second one is a trade-off since this time duration also determines the linewidth of the TOF resonance and hence the uncertainty in the determination of $\nu_{c}$. Therefore, an alternative measurement cycle with a total cycle time of only about $200 \mathrm{~ms}$ was developed. The nuclide with the shortest half-life measured until now is ${ }^{74} \mathrm{Rb}$ with $T_{1 / 2}=65 \mathrm{~ms}$ [75]. The recent efficiency gain will allow to go down to half-lives in 
the range of 20-30 ms. Further improvements may allow us to access even very short-lived and very light nuclei like ${ }^{11} \mathrm{Li}\left(T_{1 / 2}=8.9 \mathrm{~ms}\right)$ for high-precision Penning trap mass measurements.

\subsection{Resolving power}

High-accuracy mass measurements with Penning traps require clean beams to avoid systematic errors in the mass determination arising from a Coulomb interaction of different ion species (with the same mass number A, i.e. isobars, molecules or isomers) in the trap. To this end, in the preparation Penning trap, a mass-selective cooling technique is employed [25] at ISOLTRAP. A massresolving power of $10^{4}-10^{5}$ can be achieved, which is sufficient to resolve and separate isobaric contaminants even close to stability, provided the ratio of the unwanted-towanted species is below $\approx 300$. For higher ratios in the preparation trap, space charge effects of the unwanted ion species restrict the buffer gas cooling technique, as described in sect. 3.7. The resolving power in the preparation trap is strongly correlated to the buffer gas pressure. A lower pressure results in a higher resolving power provided the pressure is enough to cool the ions within an acceptable time. Thus, the duration of the preparation of a clean beam is a compromise between the half-life of the radionuclide of interest and the required cleaning.

An important issue in direct mass measurements is to resolve isomeric states since nearly one third of the nuclides in the nuclear chart have long-lived isomeric states with, in many cases, unknown excitation energies. Thus, a very high resolving power is required. Empirically, the resolving power is found to be [79]

$$
R=m / \Delta m_{\mathrm{FWHM}}=\nu_{c} / \Delta \nu_{c} \approx 1.25 \cdot \nu_{c} \cdot T_{\mathrm{q}},
$$

where $\Delta \nu_{c}$ is the FWHM of the resonance. A resolving power of $R \approx 10^{6}$ is routinely achieved in the precision Penning trap for ions with mass number 100 and with an excitation time of $T_{\mathrm{q}}=1 \mathrm{~s}$, as typically used in on-line experiments.

The dipolar cleaning procedure described in sect. 4.2 is often adopted to get rid of isomers which cannot be resolved by rf-excitation in the preparation trap. One such example is the set of mass measurements performed on the isomeric and the ground state of ${ }^{68} \mathrm{Cu}$ [80]. It has a low-lying isomeric state $6^{-}(E=721.6 \mathrm{keV})$ while its ground state is $1^{+}$. In fig. 33(a), the time-of-flight resonance is shown for a mixture of both states as delivered by ISOLDE. To avoid shifts in the determination of the cyclotron frequency due to the presence of contaminants (here the isomers), a dipolar rf-excitation at the reduced cyclotron frequency of the unwanted isomer was applied. It resulted in an isolated state, either the ground or the isomeric state, yielding only one resonance as shown in figs. 33 (b) and (c). It shows the strength of this cleaning procedure adopted in the precision trap at ISOLTRAP to prepare isomerically pure ion ensemble. This possibility along with laser and decay spectroscopy measurements

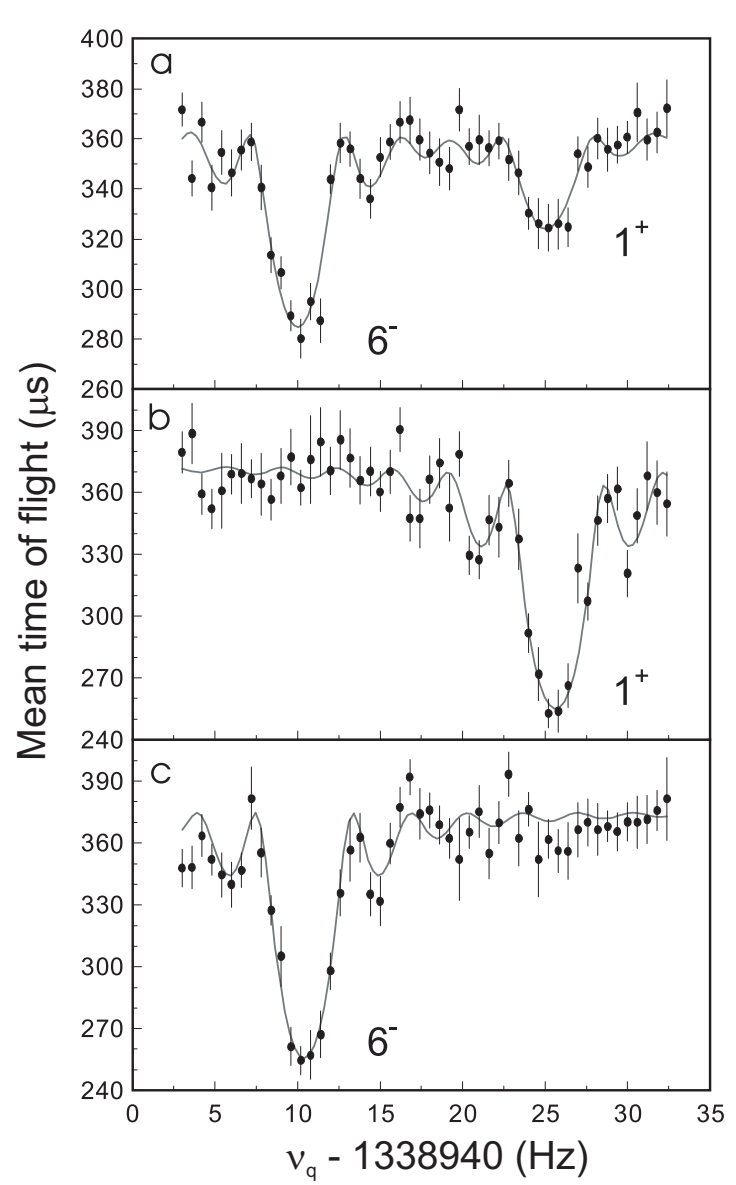

Fig. 33. The time-of-flight (TOF) resonance of ${ }^{68} \mathrm{Cu}^{+}[80]$ for (a) both the isomeric $6^{+}$and the ground $1^{-}$state, (b) the isolated ground state while the isomeric state is cleaned by dipolar reduced cyclotron excitation, and (c) the isolated isomeric state while the ground state is cleaned. In all three spectra the solid line is a fit of the theoretical function to the data [45].

recently allowed to solve the state assignment problem of ${ }^{70} \mathrm{Cu}[81]$ and the resolution of a low-lying isomeric state in ${ }^{187} \mathrm{~Pb}$ with an excitation energy of only $33 \mathrm{keV}$ [82] by ISOLTRAP

\subsection{In-trap decay}

ISOLTRAP has the capability to measure masses of all nuclides produced at ISOLDE with half-lives $\geq 50 \mathrm{~ms}$ and a production rate $\geq 100$ ions per proton pulse. These limits are given mainly by the amounts of contaminations, the length of the measurement (preparation) cycle, and the total efficiency of the present setup. Also the masses of nuclides of refractory elements can be measured which cannot directly be delivered by ISOLDE, but rather can be produced via beta decay of stored ions in the purification Penning trap. Recently, mass spectrometry with intrap decay products has been demonstrated, by which in principle masses of isotopes of refractory elements can be 
measured after they are produced by $\beta$-decay of their nonrefractory mother in the ISOLTRAP preparation trap. The pilot experiment at ISOLTRAP was performed by storing ${ }^{37} \mathrm{~K}^{+}$with a half-life of $1.2 \mathrm{~s}$ in the preparation trap for a few seconds until most of the stored ions decayed into ${ }^{37} \mathrm{Ar}^{+}$(the daughter nucleus). These ${ }^{37} \mathrm{Ar}$ ions were then transferred to the precision trap where their mass was determined [83].

\subsection{Accuracy}

Until 2002 the combined uncertainty was estimated conservatively to be $\delta m / m \approx 1 \cdot 10^{-7}$ [14], which includes such uncertainties as caused by inhomogeneities in the magnetic and electric fields (see sect. 2.6), by magnetic-field drifts or fluctuations, and the mass-dependent systematic uncertainty stemming from the difference in mass between the ion of interest and the reference ion. However, already in 1997 it was stated that an uncertainty of $3 \cdot 10^{-8}$ should be possible [84].

The carbon cluster cross-reference measurements [46, 59] allowed the study of the various contributions of different systematic effects to the combined uncertainty of a frequency ratio determination (see sect. 5.2). This investigation yielded the relative mass-dependent cyclotron frequency ratio shift given by eq. (31) which is corrected as well as added to the final uncertainty. Once all known effects are taken into account, the remaining uncertainty is found to be $8 \cdot 10^{-9}$. This also represents the current limit of the accuracy [59]. A better understanding of the setup and further developments can push even further down the accuracy limit, as it was recently observed from a mass evaluation around $A=22[73,78]$.

Yet another development in recent years towards achieving higher precision has been the implementation of the separated oscillatory field method for TOF-ICR detection, as originally developed by Ramsey [85,86] for molecular beam spectroscopy. The advantage of the Ramsey technique is the reduction of the linewidth along with more pronounced sidebands, leading to a more precise determination of the resonance frequency (see [87] and references therein). We have implemented the same technique for the ion's cyclotron frequency determination [88, 89]. In fig. 34 two time-of-flight cyclotron resonances of ${ }^{39} \mathrm{~K}^{+}$are shown, each with a total excitation time of $1.2 \mathrm{~s}$. For both resonances the total number of recorded ions has been 2500. The resonance frequency using the conventional excitation scheme is determined to $\nu_{c}=$ $2331373.6426(76) \mathrm{Hz}$ and for the Ramsey type of excitation a resonance frequency of $\nu_{c}=2331373.6397(21) \mathrm{Hz}$ is obtained. In these measurements the magnetic-field drift is not accounted for and hence a slight difference in the frequencies are obtained. The example shows that the Ramsey technique allows the measurement of the cyclotron frequency a factor of three more precise than the single pulse rf-excitation for the chosen measurement scheme. A first on-line mass measurement was performed on the short-lived nuclide ${ }^{38} \mathrm{Ca}[90]$.
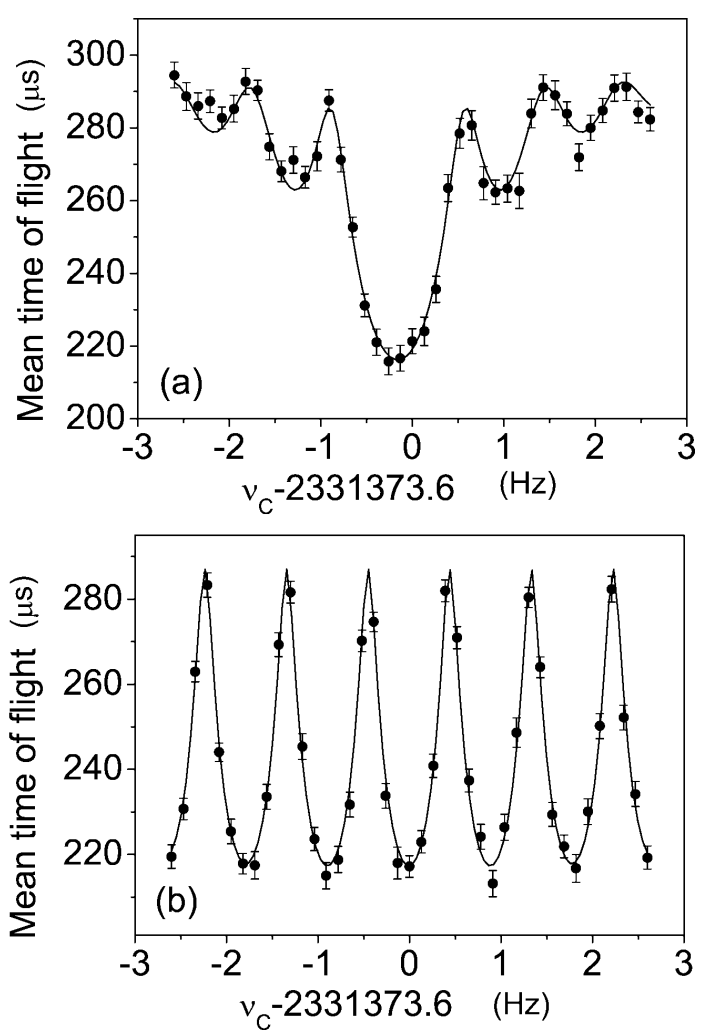

Fig. 34. The measured mean time of flight as a function of the quadrupolar excitation frequency with the theoretical curve fitted to the data (solid line). Here ${ }^{39} \mathrm{~K}^{+}$ions from the stable alkali ion source were used. (a) Resonance of the conventional one-pulse excitation scheme with $1.2 \mathrm{~s}$ excitation time; (b) a two-pulse excitation scheme with two times 100 ms excitation and $1 \mathrm{~s}$ waiting time.

\subsection{Conclusion and outlook}

So far ISOLTRAP has measured the masses of about 300 nuclides which are listed in table 6 . Table 7 compiles those data which are presently being evaluated or in the publication procedure. The uncertainties of these measurements vary between $10^{-7}$ to $10^{-8}$, thereby allowing to investigate different physics problems.

Apart from the ongoing measurement plans at ISOLTRAP, we want to push the limit of accuracy and the efficiency further. To this end, higher charge states of the ions and/or excitations with higher harmonics (like octupolar) are presently under consideration [91,92]. One way to achieve a higher charge state will be to use an electron beam ion trap (EBIT) as planned for TITAN [22].

At present elements with high electron affinity like the halogens can only be measured in some chemical compound form (if at all) since the present ISOLTRAP is designed for capturing positive ions. By changing the polarity of the transfer and trapping potentials it may soon be possible to trap anionic ions for direct mass measurements. 
Table 6. Stable and unstable nuclides whose mass has been measured at ISOLTRAP.

\begin{tabular}{lll}
\hline Element & Mass numbers & References \\
\hline $\mathrm{Ne}$ & $18,19,23,24$ & {$[93]$} \\
$\mathrm{Na}$ & 21,22 & {$[73]$} \\
$\mathrm{Mg}$ & 22 & {$[73]$} \\
$\mathrm{Ar}$ & $32-34,42-45$ & {$[65,94,95]$} \\
$\mathrm{Cr}$ & 56,57 & {$[96]$} \\
$\mathrm{Mn}$ & 56,57 & {$[97]$} \\
$\mathrm{Ni}$ & $57,60,64-69$ & {$[98]$} \\
$\mathrm{Ga}$ & $63-65,68-78$ & {$[98]$} \\
$\mathrm{Cu}$ & $65-69,68 \mathrm{~m}, 70,70 \mathrm{~m}, 70 \mathrm{n}, 71-74,76$ & {$[80,81,98]$} \\
$\mathrm{Kr}$ & $72-78,80,82,84,86,88-95$ & {$[99,100,95,101]$} \\
$\mathrm{Rb}$ & $74-81,82 \mathrm{~m}, 83,84,86-89,90 \mathrm{~m}, 91-94$ & {$[95,102,103]$} \\
$\mathrm{Sr}$ & $76-83,86-88,91-95$ & {$[102-105]$} \\
$\mathrm{Sn}$ & $124,129,130,130 \mathrm{~m}, 131,132$ & {$[105]$} \\
$\mathrm{Xe}$ & $114-123,126,136$ & {$[106,107]$} \\
$\mathrm{Cs}$ & $117-122,122 \mathrm{~m}, 123-132,134-142$ & {$[108,109]$} \\
$\mathrm{Ba}$ & $123-128,130,131,138-144$ & {$[109,110,97]$} \\
$\mathrm{Ce}$ & $132-134$ & {$[111]$} \\
$\mathrm{Nd}$ & $130,132,134-138$ & {$[110,112]$} \\
$\mathrm{Pm}$ & $136-139,140 \mathrm{~m}, 141,143$ & {$[110]$} \\
$\mathrm{Sm}$ & $136-141,141 \mathrm{~m}, 142,143$ & {$[110]$} \\
$\mathrm{Eu}$ & $139,141,142 \mathrm{~m}, 143-149,151,153$ & {$[110,79,112]$} \\
$\mathrm{Dy}$ & $148,149,154$ & {$[110,79,112]$} \\
$\mathrm{Ho}$ & 150 & {$[110]$} \\
$\mathrm{Tm}$ & 165 & {$[79]$} \\
$\mathrm{Yb}$ & $158-164$ & {$[79]$} \\
$\mathrm{Hg}$ & $179-188,190-197,185 \mathrm{~m}, 187 \mathrm{~m}, 189 \mathrm{~m}, 191 \mathrm{~m}, 193 \mathrm{~m}, 197 \mathrm{~m}, 200$ & {$[113]$} \\
$\mathrm{Pb}$ & $187,187 \mathrm{~m}, 196,198$ & {$[113,82]$} \\
$\mathrm{Bi}$ & 197 & {$[113]$} \\
$\mathrm{Po}$ & 198 & {$[113]$} \\
$\mathrm{At}$ & 203 & {$[113]$} \\
$\mathrm{Fr}$ & $209-212,221,222,230$ & {$[114,115]$} \\
$\mathrm{Ra}$ & $226,229-232$ & {$[109,114,115]$} \\
$\mathrm{K}$ & $35-38,43-46$ & {$[116]$} \\
\hline & &
\end{tabular}

Table 7. Stable and unstable nuclides whose mass has been measured at ISOLTRAP but not yet published.

\begin{tabular}{lll}
\hline Element & Mass numbers & Year of measurement \\
\hline $\mathrm{Ne}$ & 17 & 2004 \\
$\mathrm{Al}$ & 26,27 & 2006 \\
$\mathrm{Ca}$ & 38,39 & 2006 \\
$\mathrm{Mn}$ & $58 \mathrm{~m}, 59-63$ & 2003,2006 \\
$\mathrm{Fe}$ & $61-63$ & 2006 \\
$\mathrm{Zn}$ & $71 \mathrm{~m}, 72-81$ & 2005 \\
$\mathrm{Se}$ & $70-73$ & 2002 \\
$\mathrm{Br}$ & $72-75$ & 2002 \\
$\mathrm{Ag}$ & $98-101,103,112,114,116,118 \mathrm{~m}, 120,121$ & 2002,2006 \\
$\mathrm{Cd}$ & $114,120,124,126$ & 2005,2006 \\
$\mathrm{Sn}$ & $127,128 \mathrm{~m}, 133,134$ & 2004 \\
$\mathrm{Cs}$ & 145,147 & 2002 \\
$\mathrm{Ba}$ & 130 & 2003 \\
$\mathrm{Tl}$ & $181,183,186 \mathrm{~m}, 187,196$ & 2002 \\
$\mathrm{~Pb}$ & 197,208 & 2002 \\
$\mathrm{Bi}$ & $190-196,215,216$ & 2002 \\
$\mathrm{Fr}$ & $203,205,229$ & 2002 \\
$\mathrm{Ra}$ & 214 & 2002 \\
\hline
\end{tabular}


We thank P. Delahaye, M. Dworschak, C. Guénaut, H. Raimbault-Hartmann, A. Kohl, M. König, M. Kuckein, M. Marie-Jeanne, D. Lunney, R.B. Moore, D. Rodríguez, E. Sauvan, G. Sikler, H. Stolzenberg, J. Szerypo, and C. Weber for their contributions in developing the ISOLTRAP mass spectrometer to the present performance. This work was supported by the European Commission within the EUROTRAPS network under contract number ERBFM RXCT97-0144, the RTD project EXOTRAPS under contract number HPRI-CT-199800018, the NIPNET network under contract number HPRICT-2001-50034, Marie Curie foundation with number HPMTCT-2000-00197 and by the Helmholtz Association of National Research Centres (HGF) under contract number VH-NG-037. We would also like to thank BMBF for the financial support under the contract numbers 06MZ962I, 06LM968, 06GF151, and 06MZ215.

Note added in proofs: The mass of Li-11 has recently been measured at TITAN, TRIUMF, Vancouver.

\section{References}

1. G. Audi et al., Nucl. Phys. A 729, 337 (2003).

2. K. Blaum, Phys. Rep. 425, 1 (2006).

3. D. Lunney et al., Rev. Mod. Phys. 75, 1021 (2003).

4. M. Bender et al., Rev. Mod. Phys. 75, 121 (2003).

5. G.A. Lalazissis et al., Phys. Rev. C 58, 1467 (1998).

6. S. Hofmann et al., Rev. Mod. Phys. 72, 733 (2000).

7. G.R. Henry, Phys. Rev. 176, 1126 (1968).

8. E. Caurier et al., Rev. Mod. Phys. 77, 427 (2005).

9. R.B. Cakirli et al., Phys. Rev. Lett. 94, 092501 (2005).

10. R.B. Cakirli et al., Phys. Rev. Lett. 95, 119903 (2005).

11. S. Bishop et al., Phys. Rev. Lett. 90, 162501 (2003).

12. J. Hardy, I.S. Towner, Phys. Rev. Lett. 94, 092502 (2005).

13. J. Hardy et al., Phys. Rev. C 71, 055501 (2005).

14. G. Bollen et al., Nucl. Instrum. Methods A 368, 675 (1996).

15. F. Herfurth et al., J. Phys. B 36, 931 (2003).

16. K. Blaum et al., Nucl. Instrum. Methods B 204, 478 (2003).

17. H.-J. Kluge et al., Nucl. Phys. A 746, 200c (2004).

18. G. Bollen et al., Nucl. Instrum. Methods A 532, 203 (2004).

19. J. Clark et al., Nucl. Instrum. Methods B 204, 487 (2003).

20. A. Jokinen et al., Int. J. Mass Spectrom. 251, 204 (2006).

21. M. Block et al., Eur. Phys. J. A 25, 49 (2005).

22. J. Dilling et al., Int. J. Mass Spectrom. 251, 198 (2006).

23. H. Schnatz et al., Nucl. Instrum. Methods A 251, 17 (1986).

24. G. Bollen et al., J. Appl. Phys. 68, 4355 (1990).

25. G. Savard et al., Phys. Lett. A 158, 247 (1991).

26. K. Blaum et al., J. Phys. B 36, 921 (2003).

27. F. Herfurth et al., Nucl. Instrum. Methods A 469, 254 (2001).

28. E. Kugler, Hyperfine Interact. 129, 23 (2000).

29. W. Paul et al., Z. Naturforsch. 8a, 448 (1953).

30. H.G. Dehmelt, Adv. At. Mol. Phys. 3, 53 (1967).

31. W. Paul, Rev. Mod. Phys. 62, 531 (1990).

32. H. Dehmelt, Rev. Mod. Phys. 62, 525 (1990).

33. P.H. Dawson, Quadrupole Mass Spectrometry and its applications (Elsevier, Amsterdam, 1995).
34. P.K. Ghosh, Ion traps (Oxford University Press, New York, 1995).

35. K. Blaum et al., Int. J. Mass Spectrom. 181, 67 (1998).

36. A. Nieminen et al., Nucl. Instrum. Methods A 469, 244 (2001).

37. L.S. Brown, G. Gabrielse, Rev. Mod. Phys. 58, 233 (1986).

38. M. Kretzschmar, Eur. J. Phys. 12, 240 (1991).

39. S. Schwarz, Nucl. Instrum. Methods A 566, 233 (2006).

40. E.W. McDaniel, The mobility and diffusion of ions in gases (Wiley, 1973).

41. A. Kellerbauer et al., Nucl. Phys. A 701, 565c (2002).

42. H. Raimbault-Hartmann et al., Nucl. Instrum. Methods B 126, 382 (1997).

43. F. Bloch, Physica 19, 821 (1953).

44. G. Gräff et al., Z. Phys. A 297, 35 (1980).

45. M. König et al., Int. J. Mass Spectrom. Ion Process. 142, 95 (1995)

46. K. Blaum et al., Eur. Phys. J. A 15, 254 (2002).

47. K. Blaum et al., Anal. Bioanal. Chem. 377, 1133 (2003).

48. R.S. Van Dyck jr. et al., Phys. Rev. A 40, 6308 (1989).

49. E.A. Cornell et al., Phys. Rev. Lett. 63, 1674 (1989).

50. W. Jhe et al., Phys. Scr. 46, 264 (1992).

51. G. Bollen et al., Phys. Scr. 46, 581 (1992).

52. I. Bergström et al., Nucl. Instrum. Methods A 487, 618 (2002).

53. K. Jungmann et al., Phys. Rev. A 36, 3451 (1987).

54. J.B. Jeffries et al., Int. J. Mass Spectrom. Ion Process. 54, 169 (1983).

55. G. Bollen et al., Phys. Rev. C 46, R2140 (1992).

56. G. Gabrielse et al., Hyperfine Interact. 81, 5 (1993).

57. S. Rainville et al., Science 303, 334 (2004).

58. D.J. Wineland et al., J. Appl. Phys. 46, 919 (1975).

59. A. Kellerbauer et al., Eur. Phys. J. D 22, 53 (2003).

60. G. Bollen, Hyperfine Interact. 38, 793 (1987).

61. H.-J. Kluge, Phys. Scr. T 22, 85 (1988).

62. M. Lindroos, Nucl. Instrum. Methods B 205, 730 (2003).

63. U. Köster et al., Nucl. Instrum. Methods B 204, 347 (2003).

64. C. Scheidenberger et al., Nucl. Phys. A 701, 574c (2002).

65. K. Blaum et al., Eur. Phys. J. D 24, 145 (2003).

66. L. Schweikhard et al., Eur. J. Mass Spectrom. 11, 457 (2005).

67. S. Schwarz, PhD Thesis, Johannes Gutenberg University, Mainz, ISBN 3-8288-0735-6 (1999).

68. A. Kellerbauer et al., Nucl. Instrum. Methods A 469, 276 (2001).

69. D. Beck et al., Eur. Phys. J. A 8, 307 (2000).

70. M.M. Jeanne et al., submitted to Nucl. Instrum. Methods (2007) arXiv e-print: physics/0701224.

71. C. Yazidjian et al., Hyperfine Interact. 173, 181 (2007).

72. D. Beck et al., Nucl. Instrum. Methods A 527, 567 (2004).

73. M. Mukherjee et al., Phys. Rev. Lett. 93, 150801 (2004).

74. K. Blaum et al., Phys. Rev. Lett. 91, 260801 (2003).

75. A. Kellerbauer et al., Phys. Rev. Lett. 93, 072502 (2004).

76. C. Guénaut, PhD Thesis, Univeristé Paris XI, Orsay, http://tel.archives-ouvertes.fr/tel-00011595 (2005).

77. C. Guénaut et al., submitted to Int. J. Mass Spectrom. (2006).

78. M. Mukherjee et al., Mass measurements and evaluation around $A=22$, this issue.

79. G. Bollen, Nucl. Phys. A 693, 3 (2001). 
80. K. Blaum et al., Europhys. Lett. 67, 586 (2004).

81. J. Van Roosbroeck et al., Phys. Rev. Lett. 92, 11250 (2004).

82. C. Weber et al., Phys. Lett. A 347, 81 (2005).

83. A. Herlert et al., New J. Phys. 7, 44 (2005).

84. D. Beck et al., Nucl. Instrum. Methods B 126, 374 (1997).

85. I.I. Rabi et al., Phys. Rev. 53, 318 (1938).

86. I.I. Rabi et al., Phys. Rev. 55, 526 (1939).

87. N.F. Ramsey et al., Rev. Mod. Phys. 62, 541 (1990).

88. M. Kretzschmar et al., Int. J. Mass Spectrom. 264, 122 (2007).

89. S. George et al., Int. J. Mass Spectrom. 264, 110 (2007).

90. S. George et al., Phys. Rev. Lett. 98, 162501 (2007).

91. R. Ringle et al., Int. J. Mass Spectrom. 262, 33 (2007).

92. S. Eliseev et al., Int. J. Mass Spectrom. 262, 45 (2007).

93. K. Blaum et al., Nucl. Phys. A 746, 305c (2004).

94. F. Herfurth et al., Phys. Rev. Lett. 87, 142501 (2001).

95. F. Herfurth et al., Eur. Phys. J. A 15, 17 (2002).

96. C. Guénaut et al., J. Phys. G 31, S1765 (2005).

97. C. Guénaut et al., Eur. Phys. J. A 25, S1.35 (2005).
98. C. Guénaut et al., Phys. Rev. C 75, 044303 (2007).

99. D. Rodríguez et al., Phys. Rev. Lett. 93, 161104 (2004).

100. P. Delahaye et al., Phys. Rev. C 74, 034331 (2006).

101. D. Rodríguez et al., Nucl. Phys. A 769, 1 (2006).

102. H. Raimbault-Hartmann et al., Nucl. Phys. A 706, 3 (2002).

103. T. Otto et al., Nucl. Phys. A 567, 281 (1994).

104. F. Herfurth et al., Nucl. Phys. A 746, 487c (2004).

105. G. Sikler et al., Nucl. Phys. A 763, 45 (2005).

106. J. Dilling et al., Eur. Phys. J. A 22, 163 (2004).

107. A. Herlert et al., Int. J. Mass Spectrom. 251, 131 (2006).

108. H. Stolzenberg et al., Phys. Rep. Lett. 65, 3104 (1990).

109. F. Ames et al., Nucl. Phys. A 651, 3 (1999).

110. D. Beck et al., Nucl. Phys. A 626, 343c (1997).

111. G. Bollen et al., Hyperfine Interact. 132, 215 (2001).

112. D. Beck et al., Eur. Phys. J. A 8, 307 (2002).

113. S. Schwarz et al., Nucl. Phys. A 693, 533 (2001).

114. G. Bollen et al., J. Mod. Opt. 39, 257 (1992).

115. F. Herfurth et al., Eur. Phys. J. A 25, s01, 17 (2005).

116. C. Yazidjian et al., Phys. Rev. C 76, 024308 (2007). 